\title{
Boundary stabilization of the linear elastodynamic system by a Lyapunov-type method
}

\author{
Rabah BEY(1), Amar Heminna ${ }^{(2)}$ and Jean-Pierre LoHÉAC ${ }^{(1)}$ \\ (1) École Central de Lyon \\ Département de Mathématiques et Informatique - B.P. 163 \\ C.N.R.S. U.M.R. 5585 \\ 69131 Écully Cedex, France \\ Jean-Pierre.Loheac@ec-Iyon.fr \\ (2) U.S.T.H.B., \\ Institut de Mathématiques \\ B.P. 32 \\ El-Alia \\ 16111 Alger, Algeria
}

Recibido: 23 de Noviembre de 2000

Aceptado: 4 de Junio de 2003

\begin{abstract}
We propose a direct approach to obtain the boundary stabilization of the isotropic linear elastodynamic system by a "natural" feedback; this method uses local coordinates in the expression of boundary integrals as a main tool. It leads to an explicit decay rate of the energy function and requires weak geometrical conditions: for example, the spacial domain can be the difference of two star-shaped sets.
\end{abstract}

2000 Mathematics Subject Classification: 93D15, 74B05, 93B03, 93B05

Key words: Elastodynamic system, boundary stabilization, feedback

\section{Introduction}

In this paper, we present a detailed proof of a result which has been announced in [3]. This result concerns the boundary stabilization of a linear isotropic elastodynamic system. Extensions concerning the non-linear case and the anisotropic case are still in progress. 
Let $\Omega$ be a bounded connected open set of $\mathbb{R}^{3}$ such that its boundary $\Gamma$ satisfies

$$
\begin{aligned}
& \Gamma \text { is of class } \mathcal{C}^{2}, \\
& \Gamma=\Gamma_{0} \cup \Gamma_{1}, \text { with } \\
& \quad \operatorname{meas}\left(\Gamma_{0}\right) \neq 0, \quad \operatorname{meas}\left(\Gamma_{1}\right) \neq 0, \quad \bar{\Gamma}_{0} \cap \bar{\Gamma}_{1}=\emptyset .
\end{aligned}
$$

Given $\mathbf{x}$, a point of $\Gamma$, we denote by $\nu(\mathbf{x})$ the normal unit vector pointing outwards of $\Omega$.

For a regular vector field $\mathbf{v}=\left(v_{1}, v_{2}, v_{3}\right)$, we define

$$
v_{\imath, \jmath}=\partial_{\jmath} v_{\imath}, \quad \varepsilon_{\imath \jmath}(\mathbf{v})=\frac{1}{2}\left(v_{\imath, \jmath}+v_{\jmath, \imath}\right), \quad \sigma(\mathbf{v})=2 \mu \varepsilon(\mathbf{v})+\lambda \operatorname{div}(\mathbf{v}) I_{3},
$$

where $\lambda$ and $\mu$ are the Lamé coefficients and $I_{3}$ is the identity matrix of $\mathbb{R}^{3}$. Let $A$ and $B$ be two positive constants. We consider the following problem which has been introduced by Lagnese [10].

$$
\begin{cases}\mathbf{u}^{\prime \prime}-\operatorname{div}(\sigma(\mathbf{u}))=0, & \text { in } \Omega \times \mathbb{R}_{+} ; \\ \mathbf{u}=0, & \text { on } \Gamma_{0} \times \mathbb{R}_{+} \\ \sigma(\mathbf{u}) \nu+A \mathbf{u}+B \mathbf{u}^{\prime}=0, & \text { on } \Gamma_{1} \times \mathbb{R}_{+} \\ \mathbf{u}(0)=\mathbf{u}^{0}, & \text { in } \Omega ; \\ \mathbf{u}^{\prime}(0)=\mathbf{u}^{1}, & \text { in } \Omega ;\end{cases}
$$

where $\mathbf{u}^{\prime}=\partial \mathbf{u} / \partial t, \mathbf{u}^{\prime \prime}=\partial^{2} \mathbf{u} / \partial t^{2}$.

Let $\mathbb{L}^{2}(\Omega)$ (resp. $\mathbb{H}^{1}(\Omega)$ ) be the space of vector fields $\mathbf{v}$ such that every component of $\mathbf{v}$ belongs to $\mathrm{L}^{2}(\Omega)$ (resp. $\mathrm{H}^{1}(\Omega)$ ).

We introduce the space $\mathbb{H}_{\Gamma_{0}}^{1}(\Omega)=\left\{\mathbf{v} \in \mathbb{H}^{1}(\Omega) / \mathbf{v}=0\right.$, on $\left.\Gamma_{0}\right\}$ and we assume

$$
\left(\mathbf{u}^{0}, \mathbf{u}^{1}\right) \in \mathbb{H}_{\Gamma_{0}}^{1}(\Omega) \times \mathbb{L}^{2}(\Omega) .
$$

Under this assumption, by using semi-group theory, one can show that problem (2) is well-posed. The energy function associated to this problem is given by

$$
E(\mathbf{u}, t)=\frac{1}{2} \int_{\Omega}\left(\left|\mathbf{u}^{\prime}\right|^{2}+\sigma(\mathbf{u}): \varepsilon(\mathbf{u})\right) d \mathbf{x}+\frac{1}{2} \int_{\Gamma_{1}} A|\mathbf{u}|^{2} d \Gamma
$$

where $\sigma(\mathbf{u}): \varepsilon(\mathbf{u})=\operatorname{tr}(\sigma(\mathbf{u}) \varepsilon(\mathbf{u}))$.

A boundary stabilization result for this system has been proved by Alabau and Komornik in [1] under restrictive conditions concerning the shape of $\Omega$ as well as some data of the problem (in fact, $\Gamma_{1}$ is close to a sphere). This result has been extended firstly by Alabau and Komornik in [2] for the anisotropic case and secondly by Horn in [7], for the isotropic case under weaker geometrical conditions: the author used here micro-local analysis methods.

Recent works by Guesmia ([4], [5], [6]) extend results of [2] under similar restrictive geometrical conditions. 
We propose here a direct approach by using local coordinates in the expression of boundary integrals. Our conditions are only geometrical and less restrictive than in works by Alabau and Komornik or by Guesmia. Our proof is constructive and explicit decay rate estimates are obtained as done in these works. Furthermore, the reader will observe that similar conditions have been introduced by Lagnese [9] for some anisotropic linear elastodynamic systems and by Lasiecka and Triggiani [11] for the wave equation.

We assume that there exists a vector field $\mathbf{h}=\left(h_{1}, h_{2}, h_{3}\right)$ such that

$$
\mathbf{h} \in\left(\mathcal{C}^{1}(\bar{\Omega})\right)^{3}, \quad \mathbf{h} . \nu \leq 0, \text { on } \Gamma_{0}, \quad \mathbf{h} . \nu>0, \text { on } \Gamma_{1},
$$

and

$$
\begin{aligned}
\exists \alpha>0 / \quad & \forall \mathbf{v} \in\left(\mathcal{C}^{1}(\bar{\Omega})\right)^{3}, \quad \sigma_{\imath \jmath}(\mathbf{v}) h_{k, \jmath} v_{\imath, k} \geq \alpha \sigma(\mathbf{v}): \varepsilon(\mathbf{v}), \\
& \max _{\bar{\Omega}}(\operatorname{div}(\mathbf{h}))-\min _{\bar{\Omega}}(\operatorname{div}(\mathbf{h}))<2 \alpha .
\end{aligned}
$$

Under above assumptions, we obtain the following result.

Theorem 1. Assume (1). If there exists a vector field $\mathbf{h}$ satisfying (4) and (5), then there exists some constant $\omega>0$ such that for every initial data satisfying (3), the solution $\mathbf{u}$ of (2) satisfies

$$
\forall t \geq 0, \quad E(\mathbf{u}, t) \leq E(\mathbf{u}, 0) \exp (1-\omega t) .
$$

Remark 1. Choosing $\mathbf{v}=\mathbf{x}$ in the first line of assumption (5), we get $\operatorname{div}(\mathbf{h}) \geq 3 \alpha$, in $\bar{\Omega}$.

Remark 2. Since $\Omega$ is bounded and $\Gamma$ satisfies (1), $\Gamma_{1}$ is compact and using continuity of $\mathbf{h}$ and $\nu$ given in (1) and (4), we have

$$
\exists k>0: \mathbf{h}(\mathbf{x}) \cdot \nu(\mathbf{x}) \geq k, \quad \forall \mathbf{x} \in \Gamma_{1} .
$$

Remark 3. Theorem 1 remains true when replacing assumption (5) by the following weaker one.

$$
\begin{aligned}
\exists \alpha>0 / \quad \forall \mathbf{v} \in\left(\mathcal{C}^{1}(\bar{\Omega})\right)^{3}, \int_{\Omega} \sigma_{\imath \jmath}(\mathbf{v}) h_{k, \jmath} v_{\imath, k} d \mathbf{x} \geq \alpha \int_{\Omega} \sigma(\mathbf{v}): \varepsilon(\mathbf{v}) d \mathbf{x}, \\
\max _{\bar{\Omega}}(\operatorname{div}(\mathbf{h}))-\min _{\bar{\Omega}}(\operatorname{div}(\mathbf{h}))<2 \alpha, \quad \min _{\bar{\Omega}}(\operatorname{div}(\mathbf{h}))>0 .
\end{aligned}
$$

Indeed the reader will note that assumption (5) is used at the beginning of proof of Lemma 3, Subsection 3.1. Computation also holds under (6). 


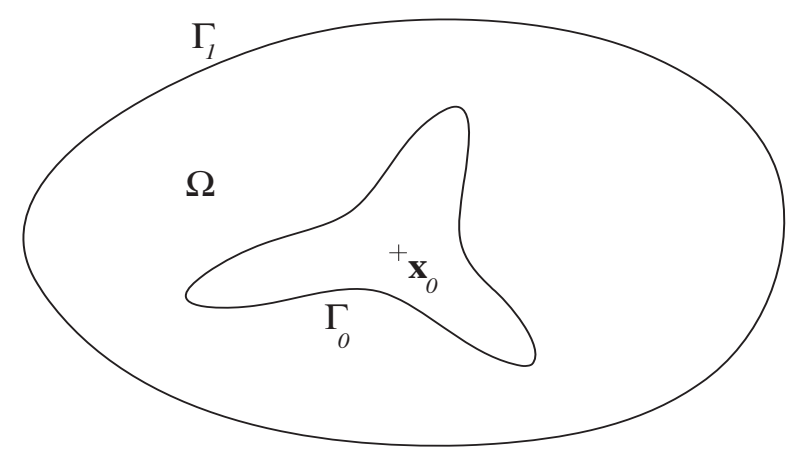

Figure 1: An example of open set $\Omega$ (Remark 4).

Remark 4. This result can be applied when $\Omega$ and its boundary $\Gamma$ satisfy (1) and

$$
\begin{aligned}
& \mathbf{h}(\mathbf{x})=\mathbf{x}-\mathbf{x}_{0},(\alpha=1), \\
& \Gamma_{0}=\{\mathbf{x} \in \Gamma / \mathbf{h}(\mathbf{x}) \cdot \nu(\mathbf{x}) \leq 0\}, \\
& \Gamma_{1}=\{\mathbf{x} \in \Gamma / \mathbf{h}(\mathbf{x}) \cdot \nu(\mathbf{x})>0\} .
\end{aligned}
$$

Especially, a possible case is: $\Omega=U_{1} \backslash U_{2}$ where $U_{1}$ is a convex open set, $U_{2}$ is a closed set, star-shaped with respect to one of its points, $\mathbf{x}_{0}$, such that $\left\{\mathbf{x}_{0}\right\} \subset U_{2} \subset U_{1}$ (see figure 1).

This case has been studied in [1], [2] [4], and [5] and for a particular shape of $\Omega\left(\Gamma_{1}\right.$ is supposed to be close to a sphere) and in [7] by means of micro-local analysis methods. Above Remark 4 can be extended in the following way.

Theorem 2. Assume (1) and suppose that, for some $\mathbf{x}_{0} \in \mathbb{R}^{3}$,

$$
\begin{aligned}
& \left(\mathbf{x}-\mathbf{x}_{0}\right) \cdot \nu(\mathbf{x}) \leq 0, \quad \text { if } \mathbf{x} \in \Gamma_{0}, \\
& \left(\mathbf{x}-\mathbf{x}_{0}\right) \cdot \nu(\mathbf{x}) \geq 0, \quad \text { if } \mathbf{x} \in \Gamma_{1} .
\end{aligned}
$$

Then there exists some constant $\omega>0$ such that for every initial data satisfying (3), the solution $\mathbf{u}$ of (2) satisfies

$$
\forall t \geq 0, \quad E(\mathbf{u}, t) \leq E(\mathbf{u}, 0) \exp (1-\omega t) .
$$

This Theorem is proved at the end of this paper. Starting from vector field $\mathbf{x}-\mathbf{x}_{0}$, we build some vector field $\mathbf{h}$ such that Theorem 1 can be applied.

This result extends previous geometrical case as follows: $\Omega=U_{1} \backslash U_{2}$ where $U_{2} \subset U_{1}$ and $U_{1}, U_{2}$ are star-shaped with respect to $\mathbf{x}_{0} \in U_{2}$ (see figure 2). 


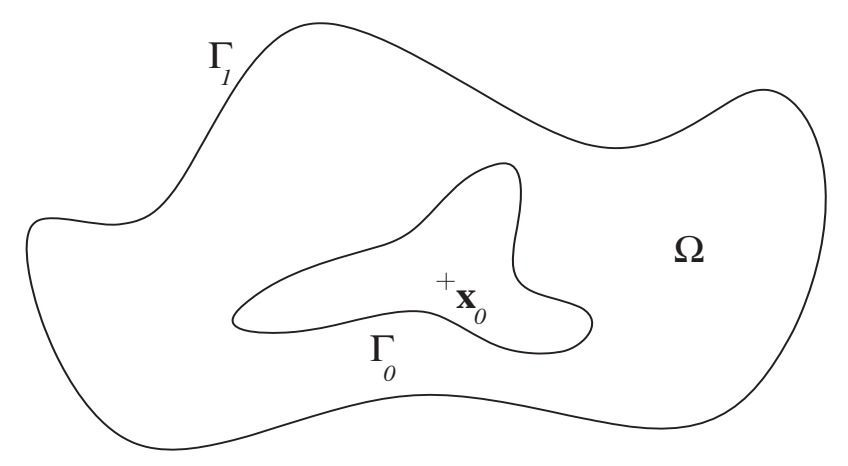

Figure 2: An example of open set $\Omega$ (Theorem 2).

Remark 5. Theorems 1 and 2 can be extended to the $n$-dimensional case. To this end, we only have to rewrite boundary integrals conveniently.

They can also be extended to the case when $A$ and $B$ belong to $\mathcal{C}^{1}\left(\Gamma_{1}\right)$.

Furthermore, Theorems 1 and 2 remain true with the following weaker assumptions concerning $A$ and $\Gamma_{0}$.

$$
\left(A=0 \quad \text { and } \operatorname{meas}\left(\Gamma_{0}\right)>0\right) \quad \text { or } \quad\left(A>0 \quad \text { and } \operatorname{meas}\left(\Gamma_{0}\right)=0\right) .
$$

This paper is mainly devoted to the proof of Theorem 1 .

After introducing some notations and definitions in Section 1, we deal with the wellposedness in Section 2 and we conclude with the stabilization in Section 3, where we describe some preliminary results and give the proof of Theorem 1. Theorem 2 is proved in Section 4.

\section{Notations and definitions}

In this paper, we use the convention of repeated indices. As usual, we write $\operatorname{tr}(\tau)=$ $\tau_{11}+\tau_{22}+\tau_{33}=\tau_{\imath \imath}, \mathbf{v} \cdot \mathbf{w}=v_{\imath} w_{\imath}, \sigma(\mathbf{v}): \varepsilon(\mathbf{v})=\sigma_{\imath \jmath}(\mathbf{v}) \varepsilon_{\imath \jmath}(\mathbf{v})$.

\subsection{Geometrical notations}

We define $\Omega, \Gamma, \nu$ as above. Since $\Gamma$ is of class $\mathcal{C}^{2}$, for every point $\mathbf{x}$ of $\Gamma$, we can build a local $\mathcal{C}^{2}$-diffeomorphism $\phi$ from an open connected subset $\hat{\Gamma}$ of $\mathbb{R}^{2}$ onto some open neighborhood of $\mathbf{x}$ in $\Gamma$. Then vectors

$$
\mathbf{a}_{\alpha}(\mathbf{x})=\frac{\partial \phi}{\partial \xi_{\alpha}}\left(\phi^{-1}(\mathbf{x})\right), \quad \alpha \in\{1,2\} ;
$$

are independent and generate the tangent plane to $\Gamma$ at $\mathbf{x}, T_{\mathbf{x}}(\Gamma)$. Furthermore, we denote by $T(\Gamma)$ the tangent bundle (see [12] and [15]). 
Then we define:

- the metric tensor $g$ related to $\phi, g_{\alpha \beta}=\mathbf{a}_{\alpha} \cdot \mathbf{a}_{\beta}, \forall(\alpha, \beta) \in\{1,2\}^{2}$;

- the inverse tensor of $g,\left(g^{\alpha \beta}\right)_{1 \leq \alpha, \beta \leq 2}$.

We denote by $\pi(\mathbf{x})$ the orthogonal projection on $T_{\mathbf{x}}(\Gamma)$ and, for a given vector field $\mathbf{v}: \bar{\Omega} \rightarrow \mathbb{R}^{3}$, we will write

$$
\begin{aligned}
\forall \mathbf{x} \in \Gamma, \quad \mathbf{v}(\mathbf{x}) & =\mathbf{v}_{T}(\mathbf{x})+v_{\nu}(\mathbf{x}) \nu(\mathbf{x}) \\
\text { with } \quad & \mathbf{v}_{T}(\mathbf{x})=\pi(\mathbf{x}) \mathbf{v}(\mathbf{x}), \quad v_{\nu}(\mathbf{x})=\mathbf{v}(\mathbf{x}) \cdot \nu(\mathbf{x}) .
\end{aligned}
$$

We denote by $\partial_{T}\left(\right.$ resp. $\left.\partial_{\nu}\right)$ the tangential (resp. normal) derivative.

If $v$ is some regular function, the transposed vector of $\partial_{T} v$ is the tangential gradient of $v$ and is denoted by $\nabla_{T} v$. We have

$$
\nabla v=\nabla_{T} v+\partial_{\nu} v \nu, \text { on } \Gamma \text {. }
$$

\subsection{Strain and stress}

If the vector field $\mathbf{v}$ is regular enough, as well as in [12] and [15], we can write

$$
\begin{aligned}
\mathrm{d} \mathbf{v}=\pi & \left(\partial_{T} \mathbf{v}_{T}\right) \pi+v_{\nu}\left(\partial_{T} \nu\right)+\left(\partial_{\nu} \mathbf{v}_{T}\right) \bar{\nu} \\
& +\nu\left(\left(\partial_{T} v_{\nu}\right)-\overline{\mathbf{v}}_{T}\left(\partial_{T} \nu\right)+\left(\partial_{\nu} v_{\nu}\right) \bar{\nu}\right), \quad \text { on } \Gamma,
\end{aligned}
$$

where $\overline{\mathbf{v}}$ (resp. $\bar{\tau}$ ) is the transposed vector (resp. matrix) of $\mathbf{v}$ (resp. $\tau$ ).

Furthermore, we can define the strain tensor $\varepsilon(\mathbf{v})$ and the stress tensor $\sigma(\mathbf{v})$ (see introduction). We have

$$
\begin{array}{ll}
\varepsilon(\mathbf{v})=\varepsilon_{T}(\mathbf{v})+\nu \overline{\varepsilon_{S}}(\mathbf{v})+\varepsilon_{S}(\mathbf{v}) \bar{\nu}+\varepsilon_{\nu}(\mathbf{v}) \nu \bar{\nu}, & \text { on } \Gamma, \\
\sigma(\mathbf{v})=\sigma_{T}(\mathbf{v})+\nu \overline{\sigma_{S}}(\mathbf{v})+\sigma_{S}(\mathbf{v}) \bar{\nu}+\sigma_{\nu}(\mathbf{v}) \nu \bar{\nu}, & \text { on } \Gamma,
\end{array}
$$

with

$$
\begin{aligned}
& 2 \varepsilon_{T}(\mathbf{v})=\pi\left(\partial_{T} \mathbf{v}_{T}\right) \pi+\pi \overline{\partial_{T} \mathbf{v}_{T}} \pi+2 v_{\nu} \partial_{T} \nu \\
& 2 \varepsilon_{S}(\mathbf{v})=\partial_{\nu} \mathbf{v}_{T}+\nabla_{T} v_{\nu}-\left(\partial_{T} \nu\right) \mathbf{v}_{T} \\
& \varepsilon_{\nu}(\mathbf{v})=\partial_{\nu} v_{\nu} \\
& \sigma_{T}(\mathbf{v})=2 \mu \varepsilon_{T}(\mathbf{v})+\lambda\left(\operatorname{tr}\left(\varepsilon_{T}(\mathbf{v})\right)+\varepsilon_{\nu}(\mathbf{v})\right) \pi \\
& \sigma_{S}(\mathbf{v})=2 \mu \varepsilon_{S}(\mathbf{v}) \\
& \sigma_{\nu}(\mathbf{v})=2 \mu \varepsilon_{\nu}(\mathbf{v})+\lambda\left(\operatorname{tr}\left(\varepsilon_{T}(\mathbf{v})\right)+\varepsilon_{\nu}(\mathbf{v})\right) .
\end{aligned}
$$

It can be observed here that terms $\varepsilon_{T}(\mathbf{v}), \sigma_{T}(\mathbf{v})$ (resp. $\varepsilon_{S}(\mathbf{v}), \sigma_{S}(\mathbf{v})$ ) correspond to some symmetric $2 \times 2$-matrices $\tilde{\varepsilon}_{S}(\mathbf{v}), \tilde{\sigma}_{T}(\mathbf{v})$ (resp. some vectors $\tilde{\varepsilon}_{S}(\mathbf{v}), \tilde{\sigma}_{S}(\mathbf{v})$ of dimension 2) such that in some orthogonal basis $\left(\tau_{1}, \tau_{2}, \nu\right)$ where $\tau_{1}, \tau_{2}$ belong to the tangent space and $\nu$ is the unit normal vector, tensors $\varepsilon(\mathbf{v})$ and $\sigma(\mathbf{v})$ are represented by matrices

$$
\left(\begin{array}{ll}
\tilde{\varepsilon}_{T}(\mathbf{v}) & \tilde{\varepsilon}_{S}(\mathbf{v}) \\
\tilde{\varepsilon}_{S}(\mathbf{v}) & \varepsilon_{\nu}(\mathbf{v})
\end{array}\right) \text { and }\left(\begin{array}{cc}
\tilde{\sigma}_{T}(\mathbf{v}) & \tilde{\sigma}_{S}(\mathbf{v}) \\
\tilde{\sigma}_{S}(\mathbf{v}) & \sigma_{\nu}(\mathbf{v})
\end{array}\right)
$$


Remark 6. Let $\mathbf{v}$ be in $\mathbb{H}^{1}(\Omega)$. From previous formulæ, we deduce

$$
\begin{aligned}
\varepsilon(\mathbf{v}): \varepsilon(\mathbf{v})= & \varepsilon_{T}(\mathbf{v}): \varepsilon_{T}(\mathbf{v})+2\left|\varepsilon_{S}(\mathbf{v})\right|^{2}+\left|\varepsilon_{\nu}(\mathbf{v})\right|^{2} \\
\sigma(\mathbf{v}): \varepsilon(\mathbf{v})= & 2 \mu\left(\varepsilon_{T}(\mathbf{v}): \varepsilon_{T}(\mathbf{v})+\left|\varepsilon_{\nu}(\mathbf{v})\right|^{2}\right)+4 \mu\left|\varepsilon_{S}(\mathbf{v})\right|^{2} \\
& +\lambda\left(\operatorname{tr}\left(\varepsilon_{T}(\mathbf{v})\right)+\varepsilon_{\nu}(\mathbf{v})\right)^{2} .
\end{aligned}
$$

We will have to consider the following vector spaces.

$\mathcal{L}_{s}\left(T_{\mathbf{x}}(\Gamma)\right)$ is the space of linear symmetric operators of $T_{\mathbf{x}}(\Gamma)$,

$\mathcal{L}_{s}(T(\Gamma))$ is the space of symmetric operators of $T(\Gamma)$.

Remark 7. $\partial_{T} \nu(\mathbf{x})$ belongs to $\mathcal{L}_{s}\left(T_{\mathbf{x}}(\Gamma)\right)$; its eigenvalues are principal curvatures of $\Gamma$ at $\mathbf{x}$.

\subsection{Some functional spaces}

Consider a tangent field $\mathbf{v}_{T}: \Gamma \rightarrow T(\Gamma)$ with $\mathbf{v}_{T}=v^{1} \mathbf{a}_{1}+v^{2} \mathbf{a}_{2}$.

We will say that $\mathbf{v}_{T}$ belongs to $\mathrm{L}^{2}(\Gamma, T(\Gamma))$ if $v^{1}$ and $v^{2}$ belong to $\mathrm{L}^{2}(\Gamma)$. In $\mathrm{L}^{2}(\Gamma, T(\Gamma))$, we define the following norm

$$
\left\|\mathbf{v}_{T}\right\|_{L^{2}(\Gamma, T(\Gamma))}=\left(\int_{\Gamma}\left|\mathbf{v}_{T}\right|^{2} d \Gamma\right)^{1 / 2}
$$

which is equivalent to the norm $\mathbf{v}_{T} \mapsto\left(\left\|v^{1}\right\|_{L^{2}(\Gamma)}^{2}+\left\|v^{2}\right\|_{L^{2}(\Gamma)}^{2}\right)^{1 / 2}$.

Similarly, $\mathbf{v}_{T}$ belongs to $\mathrm{H}^{1}(\Gamma, T(\Gamma))$ if $v^{1}$ and $v^{2}$ belong to $\mathrm{H}^{1}(\Gamma)$ and we define a norm in $\mathrm{H}^{1}(\Gamma, T(\Gamma))$ by the following formula

$$
\left\|\mathbf{v}_{T}\right\|_{\mathrm{H}^{1}(\Gamma, T(\Gamma))}=\left(\left\|v^{1}\right\|_{\mathrm{H}^{1}(\Gamma)}^{2}+\left\|v^{2}\right\|_{\mathrm{H}^{1}(\Gamma)}^{2}\right)^{1 / 2} .
$$

A field $\tau_{T}: \Gamma \rightarrow \mathcal{L}_{s}(T(\Gamma))$ belongs to $\mathrm{L}^{2}\left(\Gamma, \mathcal{L}_{s}(T(\Gamma))\right)$ if $\left(\tau_{T}: \tau_{T}\right)^{1 / 2}: \Gamma \rightarrow \mathbb{R}$ belongs to $\mathrm{L}^{2}(\Gamma)$ and we take

$$
\left\|\tau_{T}\right\|_{\mathrm{L}^{2}\left(\Gamma, \mathcal{L}_{s}(T(\Gamma))\right)}=\left\|\left(\tau_{T}: \tau_{T}\right)^{1 / 2}\right\|_{\mathrm{L}^{2}(\Gamma)}
$$

Remark 8. If $\mathbf{v}_{T} \in \mathrm{H}^{1}(\Gamma, T(\Gamma))$, then $\varepsilon_{T}\left(\mathbf{v}_{T}\right) \in \mathrm{L}^{2}\left(\Gamma, \mathcal{L}_{s}(T(\Gamma))\right)$.

Another useful space will be $\mathbb{V}=\left\{\mathbf{v} \in \mathbb{H}^{1}(\Omega) / \mathbf{v}_{T} \in \mathrm{H}^{1}(\Gamma, T(\Gamma))\right\}$ with the following norm

$$
\|\mathbf{v}\|_{\mathbb{V}}=\left(\|\mathbf{v}\|_{\mathbb{H}^{1}(\Omega)}^{2}+\left\|\mathbf{v}_{T}\right\|_{\mathrm{H}^{1}(\Gamma, T(\Gamma))}^{2}\right)^{1 / 2} .
$$

Proposition 1. By the following formula, we define in $\mathrm{H}^{1}(\Gamma, T(\Gamma))$ a norm which is equivalent to the norm given in (10)

$$
\left\|\mathbf{v}_{T}\right\|_{1}^{2}=\int_{\Gamma}\left(\left|\mathbf{v}_{T}\right|^{2}+\varepsilon_{T}\left(\mathbf{v}_{T}\right): \varepsilon_{T}\left(\mathbf{v}_{T}\right)\right) d \Gamma
$$


Proof. We only have to prove that there exists a constant $C>0$ such that

$$
\left\|\mathbf{v}_{T}\right\|_{\mathrm{H}^{1}(\Gamma, T(\Gamma))} \leq C\left\|\mathbf{v}_{T}\right\|_{1}, \quad \forall \mathbf{v}_{T} \in \mathrm{H}^{1}(\Gamma, T(\Gamma)) .
$$

Assume that such a constant does not exist.

Then there exits a sequence $\left(\mathbf{v}_{T}^{(k)}\right)$ which satisfies

$$
\begin{aligned}
& \left\|\mathbf{v}_{T}^{(k)}\right\|_{\mathrm{H}^{1}(\Gamma, T(\Gamma))}=1, \quad \forall k \in \mathbb{N} \\
& \left(\varepsilon_{T}\left(\mathbf{v}_{T}^{(k)}\right)\right) \rightarrow 0, \quad \text { in } \mathrm{L}^{2}\left(\Gamma, \mathcal{L}_{s}(T(\Gamma))\right), \\
& \left(\mathbf{v}_{T}^{(k)}\right) \rightarrow 0, \quad \text { in } \mathrm{L}^{2}(\Gamma, T(\Gamma)) .
\end{aligned}
$$

Setting $\mathbf{v}_{T}^{(k)}=v^{k 1} \mathbf{a}_{1}+v^{k 2} \mathbf{a}_{2}$ and $v_{, \beta}^{k \alpha}=\frac{\partial v^{k \alpha}}{\partial \xi_{\beta}}$, we get

$$
\left(v_{, \beta}^{k \alpha}+g^{\lambda \alpha} g_{\beta \mu} v_{, \lambda}^{k \mu}\right) \rightarrow 0, \quad \text { in } \mathrm{L}^{2}(\Gamma)
$$

With $\alpha=\beta$, we easily get $\left(v_{, 1}^{k 1}+v_{, 2}^{k 2}\right) \rightarrow 0, \quad$ in $\mathrm{L}^{2}(\Gamma)$.

We have $g_{\alpha \eta}\left(v_{, \beta}^{k \alpha}+g^{\lambda \alpha} g_{\beta \mu} v_{, \lambda}^{k \mu}\right)=g_{\alpha \eta} v_{, \beta}^{k \alpha}+g_{\beta \mu} v_{, \eta}^{k \mu}$.

This expression vanishes in $\mathrm{L}^{2}(\Gamma)$ as $k \rightarrow \infty$. We write this for $(\beta, \eta)=(1,1)$, $(\beta, \eta)=(2,2)$ and $(\beta, \eta)=(1,2)$ and we get

$$
\begin{aligned}
& \left(g_{11} v_{, 1}^{k 1}+g_{21} v_{, 1}^{k 2}\right) \rightarrow 0, \quad \text { in } \mathrm{L}^{2}(\Gamma) ; \\
& \left(g_{22} v_{, 2}^{k 2}+g_{21} v_{, 2}^{k 1}\right) \rightarrow 0, \quad \text { in } \mathrm{L}^{2}(\Gamma) ; \\
& \left(g_{11} v_{, 2}^{k 1}+g_{22} v_{, 1}^{k 2}\right) \rightarrow 0, \quad \text { in } \mathrm{L}^{2}(\Gamma) \\
& \left(v_{, 1}^{k 1}+v_{, 2}^{k 2}\right) \rightarrow 0, \quad \text { in } \mathrm{L}^{2}(\Gamma) .
\end{aligned}
$$

Set $\mathbf{w}_{T}^{(k)}=g \mathbf{v}_{T}^{(k)}=w^{k 1} \mathbf{a}_{1}+w^{k 2} \mathbf{a}_{2}$. From $\left(\mathbf{v}_{T}^{(k)}\right) \rightarrow 0$, in $\mathrm{L}^{2}(\Gamma, T(\Gamma))$ and previous computations, one can easily deduce that sequences $\left(w^{k 1}\right),\left(w^{k 1}\right),\left(w_{, 1}^{k 1}+w_{, 2}^{k 2}\right)$, $\left(w_{, 1}^{k 2}+w_{, 2}^{k 1}\right)$ vanish in $\mathrm{L}^{2}(\Gamma)$.

Thanks to Korn's inequality in $\Gamma$, we get that sequences $\left(w^{k 1}\right),\left(w^{k 2}\right)$ vanish in $\mathrm{H}^{1}(\Gamma)$.

Hence, $\left(v^{k 1}\right),\left(v^{k 2}\right)$ vanish in $\mathrm{H}^{1}(\Gamma)$ and this is impossible.

\section{Well-posedness}

By using semi-group theory, we can show that problem (2) is well-posed. This classical proof which is left to the reader leads to the following result. 
Proposition 2. If $\left(\mathbf{u}^{0}, \mathbf{u}^{1}\right)$ belongs to $\mathbb{H}_{\Gamma_{0}}^{1}(\Omega) \times \mathbb{L}^{2}(\Omega)$, then problem $(2)$ has one and only one (weak) solution $\mathbf{u}$ which satisfies

$$
\begin{aligned}
& \mathbf{u} \in \mathcal{C}^{0}\left([0,+\infty), \mathbb{H}_{\Gamma_{0}}^{1}(\Omega)\right) \cap \mathcal{C}^{1}\left([0,+\infty), \mathbb{L}^{2}(\Omega)\right) \\
& 0 \leq E(\mathbf{u}, t) \leq E(\mathbf{u}, 0), \quad \text { for almost every } t \geq 0
\end{aligned}
$$

Furthermore, if $\left(\mathbf{u}^{0}, \mathbf{u}^{1}\right)$ belongs to $\left(\mathbb{H}^{2}(\Omega) \cap \mathbb{H}_{\Gamma_{0}}^{1}(\Omega)\right) \times \mathbb{H}_{\Gamma_{0}}^{1}(\Omega)$ and if

$$
\sigma\left(\mathbf{u}^{0}\right) \cdot \nu+A \mathbf{u}^{0}+B \mathbf{u}^{1}=0, \text { on } \Gamma_{1} ;
$$

then the (strong) solution of (2) satisfies

$$
\begin{aligned}
& \left(\mathbf{u}, \mathbf{u}^{\prime}, \mathbf{u}^{\prime \prime}\right) \in \mathcal{C}^{0}\left([0,+\infty),\left(\mathbb{H}^{2}(\Omega) \cap \mathbb{H}_{\Gamma_{0}}^{1}(\Omega)\right) \times \mathbb{H}_{\Gamma_{0}}^{1}(\Omega) \times \mathbb{L}^{2}(\Omega)\right) ; \\
& \int_{\Omega}\left(\sigma\left(\mathbf{u}^{\prime}\right): \varepsilon\left(\mathbf{u}^{\prime}\right)+|\operatorname{div}(\sigma(\mathbf{u}))|^{2}\right) d \mathbf{x}+\int_{\Gamma_{1}} A\left|\mathbf{u}^{\prime}\right|^{2} d \Gamma \\
& \quad \leq \int_{\Omega}\left(\sigma\left(\mathbf{u}^{1}\right): \varepsilon\left(\mathbf{u}^{1}\right)+\left|\operatorname{div}\left(\sigma\left(\mathbf{u}^{0}\right)\right)\right|^{2}\right) d \mathbf{x}+\int_{\Gamma_{1}} A\left|\mathbf{u}^{1}\right|^{2} d \Gamma .
\end{aligned}
$$

\section{Stabilization}

Following Komornik [8], we will prove here that the energy function is exponentially decreasing with respect to time.

We recall the following fundamental result which is proved in [8].

Lemma 1. Let $E: \mathbb{R}_{+} \rightarrow \mathbb{R}_{+}$be a non-increasing function and assume that there exists $T>0$ such that

$$
\int_{t}^{\infty} E(s) d s \leq T E(t), \quad \forall t \geq 0
$$

Then we have

$$
E(t) \leq E(0) \exp \left(1-\frac{t}{T}\right), \quad \forall t \geq T
$$

First, we can prove that the energy function is non-increasing.

Proposition 3. Under assumptions (1), (3), the weak solution $\mathbf{u}$ of (2) is such that $\mathbf{u}^{\prime} \sqrt{B}$ belongs to $\mathrm{L}_{l o c}^{2}\left((0,+\infty), \mathbb{L}^{2}\left(\Gamma_{1}\right)\right)$, the energy function is non-increasing and satisfies

$$
E(\mathbf{u}, T)-E(\mathbf{u}, S)=-\int_{S}^{T} \int_{\Gamma_{1}} B\left|\mathbf{u}^{\prime}\right|^{2} d \Gamma d t, \quad 0 \leq S<T<+\infty .
$$


Proof. Assume first that $\mathbf{u}$ is a strong solution of (2) (with convenient initial data). We can write

$$
\begin{aligned}
E^{\prime}(\mathbf{u}, t) & =\int_{\Omega}\left(\mathbf{u}^{\prime} \cdot \mathbf{u}^{\prime \prime}+\sigma(\mathbf{u}): \varepsilon\left(\mathbf{u}^{\prime}\right)\right) d \mathbf{x}+\int_{\Gamma_{1}} A \mathbf{u} \cdot \mathbf{u}^{\prime} d \Gamma \\
& =\int_{\Omega}\left(\mathbf{u}^{\prime} \cdot \operatorname{div}(\sigma(\mathbf{u}))+\sigma(\mathbf{u}): \varepsilon\left(\mathbf{u}^{\prime}\right)\right) d \mathbf{x}+\int_{\Gamma_{1}} A \mathbf{u} \cdot \mathbf{u}^{\prime} d \Gamma \\
& =\int_{\Gamma_{1}}(\sigma(\mathbf{u}) \nu) \cdot \mathbf{u}^{\prime} d \Gamma+\int_{\Gamma_{1}} A \mathbf{u} \cdot \mathbf{u}^{\prime} d \Gamma \\
& =-\int_{\Gamma_{1}} B\left|\mathbf{u}^{\prime}\right|^{2} d \Gamma .
\end{aligned}
$$

We obtain the result by integrating between $S$ and $T$. A density argument completes the proof.

In order to apply Lemma 1, we have to prove some preliminary results.

\subsection{Preliminary results}

In this Subsection, we assume (1) and

$$
\begin{aligned}
& \left(\mathbf{u}^{0}, \mathbf{u}^{1}\right) \in\left(\mathbb{H}^{2}(\Omega) \cap \mathbb{H}_{\Gamma_{0}}^{1}(\Omega)\right) \times \mathbb{H}_{\Gamma_{0}}^{1}(\Omega) \\
& \sigma\left(\mathbf{u}^{0}\right) \nu+A \mathbf{u}^{0}+B \mathbf{u}^{1}=0, \text { on } \Gamma_{1}
\end{aligned}
$$

and we consider the (strong) solution of (2).

Let $\mathbf{h}$ be a vector field satisfying (4) and (5). For some positive constant $\beta$, we define

$$
M \mathbf{u}=2(\mathbf{h} . \nabla) \mathbf{u}+\beta \mathbf{u} .
$$

The value of $\beta$ will be chosen later on.

Lemma 2. The strong solution $\mathbf{u}$ of (2) satisfies

$$
\begin{aligned}
\int_{S}^{T} & \int_{\Omega}(\operatorname{div}(\mathbf{h})-\beta)\left(\left|\mathbf{u}^{\prime}\right|^{2}-\sigma(\mathbf{u}): \varepsilon(\mathbf{u})\right) d \mathbf{x} d t+2 \int_{S}^{T} \int_{\Omega} \sigma_{\imath \jmath}(\mathbf{u}) h_{k, \jmath} u_{\imath, k} d \mathbf{x} d t \\
= & -\left[\int_{\Omega} \mathbf{u}^{\prime} \cdot M \mathbf{u} d \mathbf{x}\right]_{S}^{T}+\int_{S}^{T} \int_{\Gamma} \mathbf{h} \cdot \nu\left|\mathbf{u}^{\prime}\right|^{2} d \Gamma d t \\
& +\int_{S}^{T} \int_{\Gamma}((\sigma(\mathbf{u}) \nu) \cdot M \mathbf{u}-\mathbf{h} \cdot \nu \sigma(\mathbf{u}): \varepsilon(\mathbf{u})) d \Gamma d t .
\end{aligned}
$$

Proof. We use the multipliers method (see [8], [13]). Thanks to the first equation in (2), we may write

$$
\int_{S}^{T} \int_{\Omega} \mathbf{u}^{\prime \prime} . M \mathbf{u} d \mathbf{x} d t=\int_{S}^{T} \int_{\Omega} \operatorname{div}(\sigma(\mathbf{u})) \cdot M \mathbf{u} d \mathbf{x} d t .
$$


- Consider the left-hand side.

$$
\begin{aligned}
\int_{S}^{T} \int_{\Omega} \mathbf{u}^{\prime \prime} \cdot M \mathbf{u} d \mathbf{x} d t= & {\left[\int_{\Omega} \mathbf{u}^{\prime} \cdot M \mathbf{u} d \mathbf{x}\right]_{S}^{T}-\int_{S}^{T} \int_{\Omega} \mathbf{u}^{\prime} \cdot M \mathbf{u}^{\prime} d \mathbf{x} d t } \\
= & {\left[\int_{\Omega} \mathbf{u}^{\prime} \cdot M \mathbf{u} d \mathbf{x}\right]_{S}^{T} } \\
& -2 \int_{S}^{T} \int_{\Omega} u_{\imath}^{\prime} h_{\jmath} u_{\imath, \jmath}^{\prime} d \mathbf{x} d t-\beta \int_{S}^{T} \int_{\Omega}\left|\mathbf{u}^{\prime}\right|^{2} d \mathbf{x} d t
\end{aligned}
$$

We have

$$
\begin{aligned}
2 \int_{S}^{T} \int_{\Omega} u_{\imath}^{\prime} h_{\jmath} u_{\imath, \jmath}^{\prime} d \mathbf{x} d t & =\int_{S^{T}}^{T} \int_{\Omega} h_{\jmath} \partial_{\jmath}\left(\left|\mathbf{u}^{\prime}\right|^{2}\right) d \mathbf{x} d t \\
& =\int_{S}^{T} \int_{\Gamma} \mathbf{h} \cdot \nu\left|\mathbf{u}^{\prime}\right|^{2} d \Gamma d t-\int_{S}^{T} \int_{\Omega} \operatorname{div}(\mathbf{h})\left|\mathbf{u}^{\prime}\right|^{2} d \mathbf{x} d t
\end{aligned}
$$

Hence

$$
\begin{aligned}
\int_{S}^{T} \int_{\Omega} \mathbf{u}^{\prime \prime} . M \mathbf{u} d \mathbf{x} d t= & {\left[\int_{\Omega} \mathbf{u}^{\prime} \cdot M \mathbf{u} d \mathbf{x}\right]_{S}^{T}-\int_{S}^{T} \int_{\Gamma} \mathbf{h} \cdot \nu\left|\mathbf{u}^{\prime}\right|^{2} d \Gamma d t } \\
& +\int_{S}^{T} \int_{\Omega}(\operatorname{div}(\mathbf{h})-\beta)\left|\mathbf{u}^{\prime}\right|^{2} d \mathbf{x} d t
\end{aligned}
$$

- Now, we consider the right-hand side.

$$
\begin{aligned}
\int_{S}^{T} \int_{\Omega} \operatorname{div}(\sigma(\mathbf{u})) \cdot M \mathbf{u} d \mathbf{x} d t= & 2 \int_{S}^{T} \int_{\Omega} \sigma_{\imath \jmath, \jmath}(\mathbf{u}) h_{k} u_{\imath, k} d \mathbf{x} d t \\
& +\beta \int_{S}^{T} \int_{\Omega} \mathbf{u} \cdot \operatorname{div}(\sigma(\mathbf{u})) d \mathbf{x} d t
\end{aligned}
$$

We have

$$
\begin{aligned}
\int_{S}^{T} \int_{\Omega} \sigma_{\imath \jmath, \jmath}(\mathbf{u}) h_{k} u_{\imath, k} d \mathbf{x} d t= & \int_{S}^{T} \int_{\Gamma} \sigma_{\imath \jmath}(\mathbf{u}) \nu_{\jmath} h_{k} u_{\imath, k} d \Gamma d t \\
& -\int_{S}^{T} \int_{\Omega} \sigma_{\imath \jmath}(\mathbf{u}) \partial_{\jmath}\left(h_{k} u_{\imath, k}\right) d \mathbf{x} d t \\
= & \int_{S}^{T} \int_{\Gamma}^{T} \sigma(\mathbf{u}) \nu \cdot(\mathbf{h} . \nabla) \mathbf{u} d \Gamma d t \\
& -\int_{S}^{T} \int_{\Omega} \sigma_{\imath \jmath}(\mathbf{u}) h_{k, \jmath} u_{\imath, k} d \mathbf{x} d t \\
& -\int_{S}^{T} \int_{\Omega} \sigma_{\imath \jmath}(\mathbf{u}) h_{k} u_{\imath, \jmath} d \mathbf{x} d t
\end{aligned}
$$


and

$$
\begin{aligned}
\int_{S}^{T} \int_{\Omega} \sigma_{\imath \jmath}(\mathbf{u}) h_{k} u_{\imath, \jmath k} d \mathbf{x} d t= & \frac{1}{2} \int_{S^{T}}^{T} \int_{\Omega} h_{k} \partial_{k}(\sigma(\mathbf{u}): \varepsilon(\mathbf{u})) d \mathbf{x} d t \\
= & \frac{1}{2} \int_{S}^{T} \int_{\Omega} \operatorname{div}(\mathbf{h}) \sigma(\mathbf{u}): \varepsilon(\mathbf{u}) d \mathbf{x} d t \\
& -\frac{1}{2} \int_{S}^{T} \int_{\Gamma} \mathbf{h} \cdot \nu \sigma(\mathbf{u}): \varepsilon(\mathbf{u}) d \Gamma d t
\end{aligned}
$$

Furthermore

$$
\int_{S}^{T} \int_{\Omega} \mathbf{u} \cdot \operatorname{div}(\sigma(\mathbf{u})) d \mathbf{x} d t=\int_{S}^{T} \int_{\Gamma}(\sigma(\mathbf{u}) \nu) \cdot \mathbf{u} d \Gamma d t-\int_{S}^{T} \int_{\Omega} \sigma(\mathbf{u}): \varepsilon(\mathbf{u}) d \mathbf{x} d t .
$$

Hence

$$
\begin{aligned}
\int_{S}^{T} \int_{\Omega} \operatorname{div}(\sigma(\mathbf{u})) \cdot M \mathbf{u} d \mathbf{x} d t= & \int_{S}^{T} \int_{\Omega}(\operatorname{div}(\mathbf{h})-\beta) \sigma(\mathbf{u}): \varepsilon(\mathbf{u}) d \mathbf{x} d t \\
& -2 \int_{S}^{T} \int_{\Omega} \sigma_{\imath \jmath}(\mathbf{u}) h_{k, \jmath} u_{\imath, k} d \mathbf{x} d t \\
& +\int_{S}^{T} \int_{\Gamma}^{T}((\sigma(\mathbf{u}) \nu) \cdot M \mathbf{u}-\mathbf{h} \cdot \nu \sigma(\mathbf{u}): \varepsilon(\mathbf{u})) d \Gamma d t
\end{aligned}
$$

We deduce the required result from (14), (15) and (16).

Lemma 3. There exist $\beta>0$ and $C_{1}>0$ such that

$$
\begin{aligned}
C_{1} \int_{S}^{T} E d t \leq & -\left[\int_{\Omega} \mathbf{u}^{\prime} \cdot M \mathbf{u} d \mathbf{x}\right]_{S}^{T} \\
& +\int_{S^{T}}^{T} \int_{\Gamma_{0}} \mathbf{h} \cdot \nu\left(\mu\left|\partial_{\nu} \mathbf{u}_{T}\right|^{2}+(2 \mu+\lambda)\left|\partial_{\nu} u_{\nu}\right|^{2}\right) d \Gamma d t \\
& +\int_{S^{T}}^{T} \int_{\Gamma_{1}}\left(\left(\frac{C_{1}}{2}-\beta\right) A|\mathbf{u}|^{2}-\beta B \mathbf{u} \cdot \mathbf{u}^{\prime}+\mathbf{h} \cdot \nu\left|\mathbf{u}^{\prime}\right|^{2}\right) d \Gamma d t \\
& -\int_{S}^{T} \int_{\Gamma_{1}}\left(2\left(A \mathbf{u}+B \mathbf{u}^{\prime}\right) .(\mathbf{h} . \nabla) \mathbf{u}+\mathbf{h} \cdot \nu \sigma(\mathbf{u}): \varepsilon(\mathbf{u})\right) d \Gamma d t .
\end{aligned}
$$

Proof. Lemma 2, (4) and (5) give

$$
\begin{aligned}
& \int_{S}^{T} \int_{\Omega}\left((\operatorname{div}(\mathbf{h})-\beta)\left(\left|\mathbf{u}^{\prime}\right|^{2}-\sigma(\mathbf{u}): \varepsilon(\mathbf{u})\right)+2 \alpha \sigma(\mathbf{u}): \varepsilon(\mathbf{u})\right) d \mathbf{x} d t \\
& \leq-\left[\int_{\Omega} \mathbf{u}^{\prime} \cdot M \mathbf{u} d \mathbf{x}\right]_{S}^{T}+\int_{S}^{T} \int_{\Gamma_{1}} \mathbf{h} \cdot \nu\left|\mathbf{u}^{\prime}\right|^{2} d \Gamma d t \\
& \quad+\int_{S}^{T} \int_{\Gamma}((\sigma(\mathbf{u}) \nu) \cdot M \mathbf{u}-\mathbf{h} \cdot \nu \sigma(\mathbf{u}): \varepsilon(\mathbf{u})) d \Gamma d t
\end{aligned}
$$


Since (5) holds, we can use Remark 1 and choose $\beta>0$ such that

$$
\beta<\operatorname{div}(\mathbf{h})<2 \alpha+\beta, \text { in } \bar{\Omega} ;
$$

and $C_{1}>0$ such that

$$
\begin{aligned}
C_{1} \int_{S}^{T} E d t \leq & -\left[\int_{\Omega} \mathbf{u}^{\prime} \cdot M \mathbf{u} d \mathbf{x}\right]_{S}^{T} \\
& +\int_{S^{T}}^{T} \int_{\Gamma_{1}}\left(\frac{C_{1}}{2} A|\mathbf{u}|^{2}+\mathbf{h} \cdot \nu\left|\mathbf{u}^{\prime}\right|^{2}\right) d \Gamma d t \\
& +\int_{S}^{T} \int_{\Gamma}((\sigma(\mathbf{u}) \nu) \cdot M \mathbf{u}-\mathbf{h} \cdot \nu \sigma(\mathbf{u}): \varepsilon(\mathbf{u})) d \Gamma d t .
\end{aligned}
$$

Above inequalities hold if we use assumption (6) instead of (5).

We write $\mathbf{u}=\mathbf{u}_{T}+u_{\nu} \nu$, on $\Gamma$. Because of Dirichlet boundary condition on $\Gamma_{0}$, we have

$$
M \mathbf{u}=2(\mathbf{h} . \nabla) \mathbf{u}=2 \mathbf{h} . \nu \partial_{\nu} \mathbf{u}, \text { on } \Gamma_{0} ;
$$

and, with our notations, using Remark 6 , we get

$$
\begin{aligned}
\sigma(\mathbf{u}) \nu & =\sigma_{S}(\mathbf{u})+\sigma_{\nu}(\mathbf{u}) \nu=\mu \partial_{\nu} \mathbf{u}_{T}+(2 \mu+\lambda)\left(\partial_{\nu} u_{\nu}\right) \nu, \quad \text { on } \Gamma_{0} \\
\sigma(\mathbf{u}): \varepsilon(\mathbf{u}) & =4 \mu\left|\varepsilon_{S}(\mathbf{u})\right|^{2}+(2 \mu+\lambda)\left|\varepsilon_{\nu}(\mathbf{u})\right|^{2} \\
& =\mu\left|\partial_{\nu} \mathbf{u}_{T}\right|^{2}+(2 \mu+\lambda)\left|\partial_{\nu} u_{\nu}\right|^{2}, \quad \text { on } \Gamma_{0} .
\end{aligned}
$$

Hence

$$
\begin{aligned}
& \int_{\Gamma_{0}}((\sigma(\mathbf{u}) \nu) \cdot M \mathbf{u}-\mathbf{h} \cdot \nu \sigma(\mathbf{u}): \varepsilon(\mathbf{u})) d \Gamma d t \\
& \quad=\int_{\Gamma_{0}} \mathbf{h} \cdot \nu\left(\mu\left|\partial_{\nu} \mathbf{u}_{T}\right|^{2}+(2 \mu+\lambda)\left|\partial_{\nu} u_{\nu}\right|^{2}\right) d \Gamma .
\end{aligned}
$$

Using the boundary condition on $\Gamma_{1}$, we get

$$
\begin{aligned}
& \int_{\Gamma_{1}}(\sigma(\mathbf{u}) \nu) \cdot M \mathbf{u} d \Gamma d t \\
& \quad=-\int_{\Gamma_{1}} 2\left(A \mathbf{u}+B \mathbf{u}^{\prime}\right) .(\mathbf{h} . \nabla) \mathbf{u} d \Gamma-\beta \int_{\Gamma_{1}} \mathbf{u} \cdot\left(A \mathbf{u}+B \mathbf{u}^{\prime}\right) d \Gamma
\end{aligned}
$$

We deduce the result from (18), (19) and (20).

Lemma 4. There exists $C_{2}>0$ such that

$$
\left|\int_{\Omega} \mathbf{u}^{\prime}(t) \cdot M \mathbf{u}(t) d \mathbf{x}\right| \leq C_{2} E(\mathbf{u}, t), \quad \forall t \geq 0 .
$$

Proof. Given $t \geq 0$, for every $\eta>0$, we can write

$$
\left|\int_{\Omega} \mathbf{u}^{\prime} . M \mathbf{u} d \mathbf{x}\right| \leq \frac{\eta}{2}\left\|\mathbf{u}^{\prime}\right\|_{\mathbb{L}^{2}(\Omega)}^{2}+\frac{1}{2 \eta}\|M \mathbf{u}\|_{\mathbb{L}^{2}(\Omega)}^{2} .
$$


We have

$$
\begin{aligned}
\|M \mathbf{u}\|_{\mathbb{L}^{2}(\Omega)}^{2} & =\int_{\Omega}\left(|2(\mathbf{h} . \nabla) \mathbf{u}|^{2}+\beta^{2}|\mathbf{u}|^{2}+4 \beta \mathbf{u} .(\mathbf{h} . \nabla) \mathbf{u}\right) d \mathbf{x} \\
& =\int_{\Omega}\left(|2(\mathbf{h} . \nabla) \mathbf{u}|^{2}+\beta^{2}|\mathbf{u}|^{2}+2 \beta \partial_{T}\left(|\mathbf{u}|^{2}\right) \mathbf{h}_{T}\right) d \mathbf{x} \\
& =\int_{\Omega}\left(|2(\mathbf{h} . \nabla) \mathbf{u}|^{2}+\beta(\beta-2 \operatorname{div}(\mathbf{h}))|\mathbf{u}|^{2}\right) d \mathbf{x}+2 \beta \int_{\Gamma_{1}} \mathbf{h} . \nu|\mathbf{u}|^{2} d \Gamma .
\end{aligned}
$$

Setting $R=\sup _{\bar{\Omega}}|\mathbf{h}|$, we get thanks to $(17)$

$$
\|M \mathbf{u}\|_{\mathbb{L}^{2}(\Omega)}^{2} \leq 4 R^{2} \int_{\Omega}|\nabla \mathbf{u}|^{2} d \mathbf{x}+2 \beta \int_{\Gamma_{1}} \mathbf{h} . \nu|\mathbf{u}|^{2} d \Gamma
$$

With Korn's inequality, we can find the smallest positive real number $R_{1}$ (depending on $\mathbf{h}$ and $\beta$ ) such that

$$
\begin{aligned}
4 R_{1}^{2} & \left(\int_{\Omega} \sigma(\mathbf{v}): \varepsilon(\mathbf{v}) d \mathbf{x}+\int_{\Gamma_{1}} A|\mathbf{v}|^{2} d \Gamma\right) \\
& \geq 4 R^{2} \int_{\Omega}|\nabla \mathbf{v}|^{2} d \mathbf{x}+2 \beta \int_{\Gamma_{1}} \mathbf{h} . \nu|\mathbf{v}|^{2} d \Gamma, \quad \forall \mathbf{v} \in \mathbb{H}_{\Gamma_{0}}^{1}(\Gamma) .
\end{aligned}
$$

Then we get

$$
\left|\int_{\Omega} \mathbf{u}^{\prime} \cdot M \mathbf{u} d \mathbf{x}\right| \leq \frac{\eta}{2}\left\|\mathbf{u}^{\prime}\right\|_{\mathbb{L}^{2}(\Omega)}^{2}+\frac{2 R_{1}^{2}}{\eta}\left(\int_{\Omega} \sigma(\mathbf{u}): \varepsilon(\mathbf{u}) d \mathbf{x}+\int_{\Gamma_{1}} A|\mathbf{u}|^{2} d \Gamma\right) .
$$

The choice $\eta=2 R_{1}$ gives the result with $C_{2}=2 R_{1}$.

Lemma 5. There exists $C_{3}>0$ such that, for every $\eta$ in $(0,1)$,

$$
\int_{S}^{T} \int_{\Gamma_{1}}|\mathbf{u}|^{2} d \Gamma d t \leq \frac{C_{3}}{\eta} E(\mathbf{u}, S)+\eta \int_{S}^{T} E(\mathbf{u}, t) d t, \quad 0 \leq S<T<+\infty .
$$

medskipProof. We proceed as in [8]. We define $\mathbf{z}$, depending on $t$, as follows

$$
\begin{cases}\operatorname{div}(\sigma(\mathbf{z}))=0, & \text { in } \Omega \\ \mathbf{z}=\mathbf{u}, & \text { on } \Gamma\end{cases}
$$

We have

$$
\int_{\Omega} \mathbf{z} \cdot \operatorname{div}(\sigma(\mathbf{v})) d \mathbf{x}=\int_{\Gamma_{1}} \mathbf{u} \cdot(\sigma(\mathbf{v}) \nu) d \Gamma, \quad \forall \mathbf{v} \in \mathbb{H}^{2}(\Omega) \cap \mathbb{H}_{\Gamma_{0}}^{1}(\Omega)
$$


Using the definition of the energy function and Proposition 3, we can find some positive constants $c_{1}, c_{2}, c_{1}^{\prime}, c_{2}^{\prime}$ such that

$$
\begin{aligned}
& \int_{\Omega}|\mathbf{z}|^{2} d \mathbf{x} \leq c_{1} \int_{\Gamma_{1}}|\mathbf{u}|^{2} d \Gamma \leq c_{2} E \\
& \int_{\Omega}\left|\mathbf{z}^{\prime}\right|^{2} d \mathbf{x} \leq c_{1}^{\prime} \int_{\Gamma_{1}}\left|\mathbf{u}^{\prime}\right|^{2} d \Gamma \leq c_{2}^{\prime}\left(-E^{\prime}\right) .
\end{aligned}
$$

Furthermore, we have

$$
\int_{\Omega} \sigma(\mathbf{z}): \varepsilon(\mathbf{u}-\mathbf{z}) d \mathbf{x}=-\int_{\Omega}(\mathbf{u}-\mathbf{z}) \cdot \operatorname{div}(\sigma(\mathbf{z})) d \mathbf{x}+\int_{\Gamma_{1}}(\mathbf{u}-\mathbf{z}) \cdot(\sigma(\mathbf{z}) \nu) d \Gamma=0 .
$$

Then

$$
\int_{\Omega} \sigma(\mathbf{z}): \varepsilon(\mathbf{u}) d \mathbf{x}=\int_{\Omega} \sigma(\mathbf{z}): \varepsilon(\mathbf{z}) d \mathbf{x} \geq 0
$$

From (2), we deduce

$$
\begin{aligned}
& 0=\int_{\Omega} \mathbf{z} \cdot\left(\mathbf{u}^{\prime \prime}-\operatorname{div}(\sigma(\mathbf{u})) d \mathbf{x} d t\right. \\
& =\int_{\Omega}^{\Omega} \mathbf{z} \cdot \mathbf{u}^{\prime \prime} d \mathbf{x}+\int_{\Omega} \sigma(\mathbf{z}): \varepsilon(\mathbf{u}) d \mathbf{x}-\int_{\Gamma_{1}} \mathbf{z} \cdot(\sigma(\mathbf{u}) \nu) d \Gamma \\
& =\int_{\Omega} \mathbf{z} \cdot \mathbf{u}^{\prime \prime} d \mathbf{x}+\int_{\Omega} \sigma(\mathbf{z}): \varepsilon(\mathbf{u}) d \mathbf{x}+\int_{\Gamma_{1}}^{1} \mathbf{u} \cdot\left(A \mathbf{u}+B \mathbf{u}^{\prime}\right) d \Gamma .
\end{aligned}
$$

Hence

$$
\int_{\Gamma_{1}} A|\mathbf{u}|^{2} d \Gamma \leq-\int_{\Omega} \mathbf{z} \cdot \mathbf{u}^{\prime \prime} d \mathbf{x}-\int_{\Gamma_{1}} B \mathbf{u} \cdot \mathbf{u}^{\prime} d \Gamma .
$$

For $0<S<T<\infty$, we get

$$
\begin{aligned}
\int_{S}^{T} \int_{\Gamma_{1}} A|\mathbf{u}|^{2} d \Gamma d t \leq & -\left[\int_{\Omega} \mathbf{z} \cdot \mathbf{u}^{\prime} d \mathbf{x}\right]_{S}^{T} \\
& +\int_{S}^{T} \int_{\Omega} \mathbf{z}^{\prime} \cdot \mathbf{u}^{\prime} d \mathbf{x} d t-\int_{S}^{T} \int_{\Gamma_{1}} B \mathbf{u} \cdot \mathbf{u}^{\prime} d \Gamma d t .
\end{aligned}
$$

Let $C$ be a positive constant, large enough. Using Cauchy-Schwarz inequality and above estimates, we can write for every $\theta>0$,

$$
\begin{aligned}
\int_{S}^{T} \int_{\Gamma_{1}} A|\mathbf{u}|^{2} d \Gamma d t \leq & C E(\mathbf{u}, S)+C \int_{S}^{T}\left(-E^{\prime}(\mathbf{u}, t)\right)^{1 / 2}(E(\mathbf{u}, t))^{1 / 2} d t \\
& +B \int_{S}^{T} \int_{\Gamma_{1}}|\mathbf{u}|\left|\mathbf{u}^{\prime}\right| d \Gamma d t \\
\leq & C E(\mathbf{u}, S)+\frac{C \theta}{2} \int_{S}^{T} E(\mathbf{u}, t) d t+\frac{C}{2 \theta} E(\mathbf{u}, S) \\
& +\frac{1}{2} \int_{S}^{T} \int_{\Gamma_{1}} A|\mathbf{u}|^{2} d \Gamma d t+\frac{B^{2}}{2 A} \int_{S}^{T} \int_{\Gamma_{1}}\left|\mathbf{u}^{\prime}\right|^{2} d \Gamma d t
\end{aligned}
$$


With Proposition 3, we get

$$
\begin{aligned}
\int_{S}^{T} \int_{\Gamma_{1}}|\mathbf{u}|^{2} d \Gamma d t \leq & \left(\frac{2 C}{A}+\frac{C}{A \theta}+\frac{B}{A^{2}}\right) E(\mathbf{u}, S) \\
& +\frac{C \theta}{A} \int_{S}^{T} E(\mathbf{u}, t) d t
\end{aligned}
$$

We now choose $\theta=\frac{A \eta}{C}$ and the required result follows.

\subsection{Proof of Theorem 1}

We assume (1) and $\mathbf{h}$ satisfies (4) and (5) (or (6)).

We first suppose (13) and we consider the (strong) solution $\mathbf{u}$ of (2).

The energy function is non-increasing (Proposition 3). From Lemma 4, we deduce

$$
-\left[\int_{\Omega} \mathbf{u}^{\prime} \cdot M \mathbf{u} d \mathbf{x}\right]_{S}^{T} \leq 2 C_{2} E(\mathbf{u}, S)
$$

Since h. $\nu \leq 0$ on $\Gamma_{0}$, Lemma 3 gives

$$
\begin{aligned}
C_{1} \int_{S}^{T} E d t \leq & 2 C_{2} E(\mathbf{u}, S) \\
& +\int_{S}^{T} \int_{\Gamma_{1}}\left(\left(\frac{C_{1}}{2}-\beta\right) A|\mathbf{u}|^{2}-\beta B \mathbf{u} \cdot \mathbf{u}^{\prime}+\mathbf{h} . \nu\left|\mathbf{u}^{\prime}\right|^{2}\right) d \Gamma d t \\
& -\int_{S}^{T} \int_{\Gamma_{1}}\left(2\left(A \mathbf{u}+B \mathbf{u}^{\prime}\right) .(\mathbf{h} . \nabla) \mathbf{u}+\mathbf{h} . \nu \sigma(\mathbf{u}): \varepsilon(\mathbf{u})\right) d \Gamma d t
\end{aligned}
$$

There exists $c>0$ such that

$$
\left|\int_{S}^{T} \int_{\Gamma_{1}} \beta \mathbf{u} \cdot \mathbf{u}^{\prime} d \Gamma d t\right| \leq \int_{S}^{T} \int_{\Gamma_{1}}|\mathbf{u}|^{2} d \Gamma d t+c \int_{S}^{T} \int_{\Gamma_{1}}\left|\mathbf{u}^{\prime}\right|^{2} d \Gamma d t .
$$

Hence, using Proposition 3, we can find $C_{4}>0$ and $C_{5}>0$ such that

$$
\begin{aligned}
C_{1} \int_{S}^{T} E d t \leq & C_{4} E(\mathbf{u}, S)+C_{5} \int_{S}^{T} \int_{\Gamma_{1}}|\mathbf{u}|^{2} d \Gamma d t \\
& -\int_{S}^{T} \int_{\Gamma_{1}}\left(2\left(A \mathbf{u}+B \mathbf{u}^{\prime}\right) \cdot(\mathbf{h} . \nabla) \mathbf{u}+\mathbf{h} . \nu \sigma(\mathbf{u}): \varepsilon(\mathbf{u})\right) d \Gamma d t .
\end{aligned}
$$


With Remarks 2 and 6 , this can be written

$$
\begin{aligned}
C_{1} \int_{S}^{T} E d t \leq & C_{4} E(\mathbf{u}, S)+C_{5} \int_{S}^{T} \int_{\Gamma_{1}}|\mathbf{u}|^{2} d \Gamma d t \\
& -\int_{S}^{T} \int_{\Gamma_{1}} 2\left(A \mathbf{u}+B \mathbf{u}^{\prime}\right) \cdot(\mathbf{h} . \nabla) \mathbf{u} d \Gamma d t \\
& -2 k \mu \int_{S}^{T} \int_{\Gamma_{1}}\left(\varepsilon_{T}(\mathbf{u}): \varepsilon_{T}(\mathbf{u})+\left|\varepsilon_{\nu}(\mathbf{u})\right|^{2}+2\left|\varepsilon_{S}(\mathbf{u})\right|^{2}\right) d \Gamma d t \\
& -k \lambda \int_{S}^{T} \int_{\Gamma_{1}}\left(\operatorname{tr}\left(\varepsilon_{T}(\mathbf{u})\right)+\varepsilon_{\nu}(\mathbf{u})\right)^{2} d \Gamma d t .
\end{aligned}
$$

Now, we estimate two integrals which appear in the right hand side at the second row of the above formula

- Estimate of $\mathcal{I}_{1}=\int_{S}^{T} \int_{\Gamma_{1}} 2 A \mathbf{u} .(\mathbf{h} . \nabla) \mathbf{u} d \Gamma d t$.

We denote by $C$ some positive constant which is independent of $\mathbf{u}$ and large enough.

We have $\mathbf{u} .(\mathbf{h} . \nabla) \mathbf{u}=\frac{1}{2} \mathbf{h} . \nabla\left(|\mathbf{u}|^{2}\right)$.

Setting $\mathbf{u}=\mathbf{u}_{T}+u_{\nu} \nu$ and $\mathbf{h}=\mathbf{h}_{T}+h_{\nu} \nu$, on $\Gamma_{1}$, we use (7) and we get:

$$
\mathbf{u} .(\mathbf{h} . \nabla) \mathbf{u}=\frac{1}{2} \nabla_{T}\left(|\mathbf{u}|^{2}\right) \cdot \mathbf{h}_{T}+h_{\nu} \mathbf{u}_{T} \cdot\left(\partial_{\nu} \mathbf{u}_{T}\right)+h_{\nu} u_{\nu}\left(\partial_{\nu} u_{\nu}\right), \quad \text { on } \Gamma_{1} .
$$

First, we have

$$
\int_{\Gamma_{1}} A \nabla_{T}\left(|\mathbf{u}|^{2}\right) \cdot \mathbf{h}_{T} d \Gamma d t=-\int_{\Gamma_{1}} A|\mathbf{u}|^{2} \operatorname{div}_{T}\left(\mathbf{h}_{T}\right) d \Gamma d t
$$

Hence

$$
\left|\int_{\Gamma_{1}} A \nabla_{T}\left(|\mathbf{u}|^{2}\right) \cdot \mathbf{h}_{T} d \Gamma\right| \leq C \int_{\Gamma_{1}}|\mathbf{u}|^{2} d \Gamma
$$

Using $\varepsilon_{S}(\mathbf{u})$ (see Subsection 1.2), we can write

$$
h_{\nu} \mathbf{u}_{T} \cdot \partial_{\nu} \mathbf{u}_{T}=h_{\nu} \mathbf{u}_{T} \cdot\left(2 \varepsilon_{S}(\mathbf{u})+\left(\partial_{T} \nu\right) \mathbf{u}_{T}-\nabla_{T} u_{\nu}\right) \text {, on } \Gamma_{1} .
$$

Let $\theta$ be some positive number. We can write

$$
\left|\int_{\Gamma_{1}} 4 A h_{\nu} \mathbf{u}_{T} \cdot \varepsilon_{S}(\mathbf{u}) d \Gamma\right| \leq \theta \int_{\Gamma_{1}}\left|\varepsilon_{S}(\mathbf{u})\right|^{2} d \Gamma+\frac{C}{\theta} \int_{\Gamma_{1}}\left|\mathbf{u}_{T}\right|^{2} d \Gamma .
$$

Since $h_{\nu}$ and $\partial_{T} \nu$ are bounded, we get

$$
\left|\int_{\Gamma_{1}} 2 A h_{\nu} \mathbf{u}_{T} \cdot\left(\partial_{T} \nu\right) \mathbf{u}_{T} d \Gamma\right| \leq C \int_{\Gamma_{1}}\left|\mathbf{u}_{T}\right|^{2} d \Gamma .
$$


Now, we observe

$$
\begin{aligned}
\int_{\Gamma_{1}} h_{\nu} \mathbf{u}_{T} \cdot \nabla_{T} u_{\nu} d \Gamma & =-\int_{\Gamma_{1}} u_{\nu} \underset{T}{\operatorname{div}}\left(h_{\nu} \mathbf{u}_{T}\right) d \Gamma \\
& =-\int_{\Gamma_{1}} h_{\nu} u_{\nu} \operatorname{div}_{T}\left(\mathbf{u}_{T}\right) d \Gamma-\int_{\Gamma_{1}} u_{\nu} \nabla_{T} h_{\nu} \cdot \mathbf{u}_{T} d \Gamma .
\end{aligned}
$$

Proposition 1 implies

$$
\left|\int_{\Gamma_{1}} 2 A h_{\nu} u_{\nu} \operatorname{div}_{T}\left(\mathbf{u}_{T}\right) d \Gamma\right| \leq \theta\left\|\mathbf{u}_{T}\right\|_{1}^{2}+\frac{C}{\theta} \int_{\Gamma_{1}}|\mathbf{u}|^{2} d \Gamma .
$$

Hence

$$
\left|\int_{\Gamma_{1}} 2 A h_{\nu} \mathbf{u}_{T} \cdot \nabla_{T} u_{\nu} d \Gamma\right| \leq \theta \int_{\Gamma_{1}} \varepsilon_{T}\left(\mathbf{u}_{T}\right): \varepsilon_{T}\left(\mathbf{u}_{T}\right) d \Gamma+\frac{C}{\theta} \int_{\Gamma_{1}}|\mathbf{u}|^{2} d \Gamma .
$$

We also can write

$$
\left|\int_{\Gamma_{1}} 2 A h_{\nu} u_{\nu}\left(\partial_{\nu} u_{\nu}\right) d \Gamma\right| \leq \theta \int_{\Gamma_{1}}\left|\partial_{\nu} u_{\nu}\right|^{2} d \Gamma+\frac{C}{\theta} \int_{\Gamma_{1}}|\mathbf{u}|^{2} d \Gamma .
$$

Finally, (22)-(26) give

$$
\begin{aligned}
\left|\mathcal{I}_{1}\right| \leq & \theta \int_{S}^{T} \int_{\Gamma_{1}}\left(\left|\partial_{\nu} u_{\nu}\right|^{2}+\varepsilon_{T}\left(\mathbf{u}_{T}\right): \varepsilon_{T}\left(\mathbf{u}_{T}\right)+\left|\varepsilon_{S}(\mathbf{u})\right|^{2}\right) d \Gamma d t \\
& +\frac{C}{\theta} \int_{S}^{T} \int_{\Gamma_{1}}|\mathbf{u}|^{2} d \Gamma d t
\end{aligned}
$$

We emphasize that, in (27), $\theta$ is a positive number which will be chosen later on and $C$ is a positive constant which does not depend on $\mathbf{u}$.

- Estimate of $\mathcal{I}_{2}=\int_{S}^{T} \int_{\Gamma_{1}} 2 B \mathbf{u}^{\prime} .(\mathbf{h} . \nabla) \mathbf{u} d \Gamma d t$

Here, we use (8) and we get

$$
\begin{aligned}
\mathbf{u}^{\prime} .(\mathbf{h} . \nabla) \mathbf{u}= & \overline{\mathbf{u}_{T}^{\prime}}\left(\partial_{T} \mathbf{u}_{T}\right) \mathbf{h}_{T}+u_{\nu} \overline{\mathbf{u}_{T}^{\prime}}\left(\partial_{T} \nu\right) \mathbf{h}_{T}+h_{\nu} \overline{\mathbf{u}_{T}^{\prime}} \partial_{\nu} \mathbf{u}_{T} \\
& +u_{\nu}^{\prime}\left(\partial_{T} u_{\nu}\right) \mathbf{h}_{T}-u_{\nu}^{\prime} \overline{\mathbf{u}_{T}}\left(\partial_{T} \nu\right) \mathbf{h}_{T}+u_{\nu}^{\prime}\left(\partial_{\nu} u_{\nu}\right) h_{\nu}, \quad \text { on } \Gamma_{1} .
\end{aligned}
$$

This can be written as follows

$$
\begin{aligned}
\mathbf{u}^{\prime} \cdot(\mathbf{h} . \nabla) \mathbf{u}=\mathbf{u}_{T}^{\prime} \cdot\left(\partial_{T} \mathbf{u}_{T}\right) \mathbf{h}_{T}+\left(u_{\nu} \mathbf{u}_{T}^{\prime}-u_{\nu}^{\prime} \mathbf{u}_{T}\right) \cdot\left(\partial_{T} \nu\right) \mathbf{h}_{T} & \\
& +u_{\nu}^{\prime} \nabla_{T} u_{\nu} \cdot \mathbf{h}_{T}+h_{\nu}\left(\mathbf{u}_{T}^{\prime} \cdot \partial_{\nu} \mathbf{u}_{T}+u_{\nu}^{\prime}\left(\partial_{\nu} u_{\nu}\right)\right), \quad \text { on } \Gamma_{1} .
\end{aligned}
$$

As above, since $\mathbf{h}$ and $\partial_{T} \nu$ are bounded, we get

$$
\left|\int_{\Gamma_{1}} 2 B \mathbf{u}_{T}^{\prime} \cdot\left(\partial_{T} \mathbf{u}_{T}\right) \mathbf{h}_{T} d \Gamma\right| \leq \theta\left\|\mathbf{u}_{T}\right\|_{1}^{2}+\frac{C}{\theta} \int_{\Gamma_{1}}\left|\mathbf{u}_{T}^{\prime}\right|^{2} d \Gamma
$$




$$
\left|\int_{\Gamma_{1}} 2 B\left(u_{\nu} \mathbf{u}_{T}^{\prime}-u_{\nu}^{\prime} \mathbf{u}_{T}\right) \cdot\left(\partial_{T} \nu\right) \mathbf{h}_{T} d \Gamma\right| \leq \theta \int_{\Gamma_{1}}|\mathbf{u}|^{2} d \Gamma+\frac{C}{\theta} \int_{\Gamma_{1}}\left|\mathbf{u}^{\prime}\right|^{2} d \Gamma .
$$

Under assumptions about $\Omega$, we can observe that $\Gamma_{1}$ is a compact manifold of dimension 2. So, we can build a finite number of local maps and an associated partition of unity $\left(\vartheta_{1}, \vartheta_{2}, \ldots, \vartheta_{k}\right)$. Denoting by $U_{\jmath}$ the support of $\vartheta_{\jmath}$, we can write

$$
\int_{\Gamma_{1}} 2 B u_{\nu}^{\prime} \nabla_{T} u_{\nu} \cdot \mathbf{h}_{T} d \Gamma=\sum_{\jmath=1}^{\ell} \int_{U_{\jmath}} 2 B \vartheta_{\jmath} u_{\nu}^{\prime} \nabla_{T} u_{\nu} \cdot \mathbf{h}_{T} d \Gamma .
$$

We consider one of the $\ell$ terms of the previous sum. Omitting the index $\jmath$, we denote it by $\int_{U} 2 B \vartheta u_{\nu}^{\prime} \nabla_{T} u_{\nu} \cdot \mathbf{h}_{T} d \Gamma$.

Using notations introduced in Subsection 1.1, we write $\mathbf{h}_{T}=h^{1} a_{1}+h^{2} a_{2}$. Setting $|g|=|\operatorname{det}(g)|, W=\phi^{-1}(U)$, we get

$$
\begin{aligned}
& \int_{U} 2 B \vartheta u_{\nu}^{\prime} \nabla_{T} u_{\nu} \cdot \mathbf{h}_{T} d \Gamma \\
& \quad=\int_{W} 2 B(\vartheta \circ \phi)\left(u_{\nu}^{\prime} \circ \phi\right)\left(\frac{\partial\left(u_{\nu} \circ \phi\right)}{\partial \xi_{1}} h^{1}+\frac{\partial\left(u_{\nu} \circ \phi\right)}{\partial \xi_{2}} h^{2}\right)|g|^{1 / 2} d \xi_{1} d \xi_{2} .
\end{aligned}
$$

We observe that $\vartheta \circ \phi$ is continuous and compactly supported, $v_{\nu}=u_{\nu} \circ \phi$ belongs to $\mathrm{H}^{1 / 2}(W),\left\|v_{\nu}\right\|_{\mathrm{H}^{1 / 2}(W)} \leq C\left\|u_{\nu}\right\|_{\mathrm{H}^{1 / 2}(U)}$ and we define two subsets of $W$

$$
W^{+}=\left\{\left(\xi_{1}, \xi_{2}\right) / h^{1}\left(\xi_{1}, \xi_{2}\right)>0\right\}, \quad W^{-}=\left\{\left(\xi_{1}, \xi_{2}\right) / h^{1}\left(\xi_{1}, \xi_{2}\right)<0\right\} .
$$

We have

$$
\begin{aligned}
\int_{W} 2 B(\vartheta \circ \phi) v_{\nu}^{\prime} \frac{\partial v_{\nu}}{\partial \xi_{1}} h^{1}|g|^{1 / 2} d \xi_{1} d \xi_{2} \\
=\int_{W^{+}} 2 B(\vartheta \circ \phi) v_{\nu}^{\prime} \frac{\partial v_{\nu}}{\partial \xi_{1}} h^{1}|g|^{1 / 2} d \xi_{1} d \xi_{2} \\
\quad+\int_{W^{-}} 2 B(\vartheta \circ \phi) v_{\nu}^{\prime} \frac{\partial v_{\nu}}{\partial \xi_{1}} h^{1}|g|^{1 / 2} d \xi_{1} d \xi_{2}
\end{aligned}
$$

Setting $\psi=\left((\vartheta \circ \phi) h^{1}|g|^{1 / 2}\right)^{1 / 2}$, in $W^{+}$, we can write

$$
\begin{aligned}
\int_{W^{+}} & 2 B(\vartheta \circ \phi) v_{\nu}^{\prime} \frac{\partial v_{\nu}}{\partial \xi_{1}} h^{1}|g|^{1 / 2} d \xi_{1} d \xi_{2}=\int_{W^{+}} 2 B \psi^{2} v_{\nu}^{\prime} \frac{\partial v_{\nu}}{\partial \xi_{1}} d \xi_{1} d \xi_{2} \\
= & \int_{W^{+}} 2 B \psi v_{\nu}^{\prime} \frac{\partial\left(\psi v_{\nu}\right)}{\partial \xi_{1}} d \xi_{1} d \xi_{2}-\int_{W^{+}} B \psi\left(\left|v_{\nu}\right|^{2}\right)^{\prime} \frac{\partial \psi}{\partial \xi_{1}} d \xi_{1} d \xi_{2} .
\end{aligned}
$$

We have

$$
\left|\left[\int_{W^{+}} B \psi\left|v_{\nu}\right|^{2} \frac{\partial \psi}{\partial \xi_{1}} d \xi_{1} d \xi_{2}\right]_{S}^{T}\right| \leq C E(\mathbf{u}, S) .
$$


We can observe that $\psi$ is compactly supported in $W$, and $\psi=0$, on $\partial W^{+}$. We define function $G$ by

$$
G=\psi v_{\nu}, \text { in } W^{+} \times \mathbb{R}_{+}, \quad G=0, \text { in }\left(\mathbb{R}^{2} \backslash W^{+}\right) \times \mathbb{R}_{+} .
$$

We have

$$
\int_{W^{+}} 2 B \psi v_{\nu}^{\prime} \frac{\partial\left(\psi v_{\nu}\right)}{\partial \xi_{1}} d \xi_{1} d \xi_{2}=\int_{\mathbb{R}^{2}} 2 B G^{\prime} \frac{\partial G}{\partial \xi_{1}} d \xi_{1} d \xi_{2}
$$

Let $\hat{G}$ be the Fourier transform of $G$, with respect to $\xi_{1}$. We can write

$$
\int_{W^{+}} 2 B \psi v_{\nu}^{\prime} \frac{\partial\left(\psi v_{\nu}\right)}{\partial \xi_{1}} d \xi_{1} d \xi_{2}=\int_{\mathbb{R}^{2}} 4 B \imath \pi \eta_{1} \hat{G}^{\prime} \hat{G} d \eta_{1} d \xi_{2} .
$$

This implies

$$
\int_{S}^{T} \int_{W^{+}} 2 B \psi v_{\nu}^{\prime} \frac{\partial\left(\psi v_{\nu}\right)}{\partial \xi_{1}} d \xi_{1} d \xi_{2} d t=\left[\int_{\mathbb{R}^{2}} 2 B \imath \pi \eta_{1}|\hat{G}|^{2} d \eta_{1} d \xi_{2}\right]_{S}^{T}
$$

But

$$
\left.\left|\int_{\mathbb{R}^{2}} \eta_{1}\right| \hat{G}\right|^{2} d \eta_{1} d \xi_{2} \mid \leq C_{1}\|G\|_{\mathrm{H}^{1 / 2}\left(\mathbb{R}^{2}\right)}^{2} \leq C_{2}\left\|u_{\nu}\right\|_{\mathrm{H}^{1 / 2}\left(\Gamma_{1}\right)}^{2} .
$$

Hence, using the energy function and Proposition 3, we get

$$
\left|\int_{S}^{T} \int_{W^{+}} 2 B \psi v_{\nu}^{\prime} \frac{\partial\left(\psi v_{\nu}\right)}{\partial \xi_{1}} d \xi_{1} d \xi_{2} d t\right| \leq C E(\mathbf{u}, S) .
$$

For the integral in $W^{-}$, we replace $\phi$ by $\tilde{\phi}$ such that $\tilde{\phi}\left(\xi_{1}, \xi_{2}\right)=\phi\left(-\xi_{1}, \xi_{2}\right), a_{1}$ by $-a_{1}$, $h^{1}$ by $-h^{1}$, respectively and we proceed as above. We can also get a similar result concerning the integral terms containing $h^{2}$.

Finally we obtain

$$
\left|\int_{S}^{T} \int_{\Gamma_{1}} 2 B u_{\nu}^{\prime} \nabla_{T} u_{\nu} \cdot \mathbf{h}_{T} d \Gamma d t\right| \leq C E(\mathbf{u}, S) .
$$

Using $\varepsilon_{S}(\mathbf{u})$, we get

$$
h_{\nu} \mathbf{u}_{T}^{\prime} \cdot \partial_{\nu} \mathbf{u}_{T}=h_{\nu} \mathbf{u}_{T}^{\prime} \cdot\left(2 \varepsilon_{S}(\mathbf{u})-\left(\nabla_{T} u_{\nu}\right)+\partial_{T} \nu \mathbf{u}_{T}\right), \text { on } \Gamma_{1} ;
$$

and, as well as for (23) and (24),

$$
\begin{gathered}
\left|\int_{\Gamma_{1}} 2 B h_{\nu} \mathbf{u}_{T}^{\prime} \cdot\left(\partial_{T} \nu\right) \mathbf{u}_{T} d \Gamma\right| \leq C \int_{\Gamma_{1}}\left(\left|\mathbf{u}_{T}^{\prime}\right|^{2}+\left|\mathbf{u}_{T}\right|^{2}\right) d \Gamma ; \\
\left|\int_{\Gamma_{1}} 4 B h_{\nu} \mathbf{u}_{T}^{\prime} \cdot \varepsilon_{S}(\mathbf{u}) d \Gamma\right| \leq \theta \int_{\Gamma_{1}}\left|\varepsilon_{S}(\mathbf{u})\right|^{2} d \Gamma+\frac{C}{\theta} \int_{\Gamma_{1}}\left|\mathbf{u}_{T}^{\prime}\right|^{2} d \Gamma .
\end{gathered}
$$


Now, we compute

$$
\begin{aligned}
\int_{S}^{T} & \int_{\Gamma_{1}} h_{\nu} \mathbf{u}_{T}^{\prime} \cdot \nabla_{T} u_{\nu} d \Gamma d t \\
& =\left[\int_{\Gamma_{1}} h_{\nu} \mathbf{u}_{T} \cdot \nabla_{T} u_{\nu} d \Gamma\right]_{S}^{T}-\int_{S}^{T} \int_{\Gamma_{1}} h_{\nu} \mathbf{u}_{T} \cdot\left(\nabla_{T} u_{\nu}^{\prime}\right) d \Gamma d t \\
& =\left[\int_{\Gamma_{1}} h_{\nu} \mathbf{u}_{T} \cdot \nabla_{T} u_{\nu} d \Gamma\right]_{S}^{T}+\int_{S}^{T} \int_{\Gamma_{1}} u_{\nu}^{\prime} \operatorname{div}_{T}\left(k \mathbf{u}_{T}\right) d \Gamma d t
\end{aligned}
$$

Since $\operatorname{div}_{T}\left(h_{\nu} \mathbf{u}_{T}\right)=h_{\nu} \operatorname{div}_{T}\left(\mathbf{u}_{T}\right)+\nabla_{T} h_{\nu} \cdot \mathbf{u}_{T}$, Proposition 1 gives

$$
\left|\int_{\Gamma_{1}} 2 B u_{\nu}^{\prime} \operatorname{div}_{T}\left(h_{\nu} \mathbf{u}_{T}\right) d \Gamma\right| \leq \theta\left\|\mathbf{u}_{T}\right\|_{1}^{2}+\frac{C}{\theta} \int_{\Gamma_{1}}\left|\mathbf{u}^{\prime}\right|^{2} d \Gamma .
$$

Now, we consider $\int_{\Gamma_{1}} h_{\nu} \mathbf{u}_{T} \cdot \nabla_{T} u_{\nu} d \Gamma$.

Given $t>0$, let $\zeta$ be in $\mathrm{H}^{1}\left(\Gamma_{1}\right)$ (notice that $\mathrm{H}^{1}\left(\Gamma_{1}\right)=\mathrm{H}_{0}^{1}\left(\Gamma_{1}\right)$ ) such that

$$
\zeta-\Delta_{T} \zeta=\operatorname{div}_{T}\left(\mathbf{u}_{T}\right)(t) .
$$

Since $\operatorname{div}_{T}\left(\mathbf{u}_{T}\right)(t)$ belongs to $\mathrm{H}^{-1 / 2}\left(\Gamma_{1}\right), \zeta$ satisfies

$$
\left\{\begin{array}{l}
\|\zeta\|_{\mathrm{H}^{1}\left(\Gamma_{1}\right)} \leq C\left\|\mathbf{u}_{T}(t)\right\|_{\mathrm{L}^{2}\left(\Gamma_{1}, T\left(\Gamma_{1}\right)\right)} \\
\zeta \in \mathrm{H}^{3 / 2}\left(\Gamma_{1}\right) \text { and }\|\zeta\|_{\mathrm{H}^{3 / 2}\left(\Gamma_{1}\right)} \leq C\left\|\mathbf{u}_{T}(t)\right\|_{\mathrm{H}^{1 / 2}\left(\Gamma_{1}, T\left(\Gamma_{1}\right)\right)}
\end{array}\right.
$$

Then we have

$$
\begin{aligned}
\int_{\Gamma_{1}} h_{\nu} \mathbf{u}_{T} \cdot \nabla_{T} u_{\nu} d \Gamma=-\int_{\Gamma_{1}} u_{\nu} \underset{T}{\operatorname{div}}\left(h_{\nu} \mathbf{u}_{T}\right) d \Gamma \\
=-\int_{\Gamma_{1}} u_{\nu} \nabla_{T} h_{\nu} \cdot \mathbf{u}_{T} d \Gamma-\int_{\Gamma_{1}} u_{\nu} h_{\nu} \zeta d \Gamma+\int_{\Gamma_{1}} u_{\nu} h_{\nu} \Delta_{T} \zeta d \Gamma .
\end{aligned}
$$

First, we can write thanks to (34)

$$
\begin{aligned}
& \left|\int_{\Gamma_{1}} u_{\nu} \nabla_{T} h_{\nu} \cdot \mathbf{u}_{T} d \Gamma\right| \leq C \int_{\Gamma_{1}}|\mathbf{u}|^{2} d \Gamma \\
& \left|\int_{\Gamma_{1}}^{u_{\nu}} u_{\nu} \zeta d \Gamma\right| \leq C \int_{\Gamma_{1}}\left(\left|u_{\nu}\right|^{2}+|\zeta|^{2}\right) d \Gamma \leq C \int_{\Gamma_{1}}|\mathbf{u}|^{2} d \Gamma .
\end{aligned}
$$

Secondly, we have

$$
-\int_{\Gamma_{1}} u_{\nu} h_{\nu} \Delta_{T} \zeta d \Gamma=\int_{\Gamma_{1}}\left(-\Delta_{T}\right)^{1 / 4}\left(u_{\nu} h_{\nu}\right)\left(-\Delta_{T}\right)^{3 / 4} \zeta d \Gamma ;
$$


and, again with (33),

$$
\left|\int_{\Gamma_{1}} u_{\nu} h_{\nu} \Delta \zeta d \Gamma\right| \leq C\left\|u_{\nu}\right\|_{\mathrm{H}^{1 / 2}\left(\Gamma_{1}\right)}\|\zeta\|_{\mathrm{H}^{3 / 2}\left(\Gamma_{1}\right)} \leq C\|\mathbf{u}\|_{\mathrm{H}^{1}(\Omega)}^{2} .
$$

Hence

$$
\left|\int_{\Gamma_{1}} h_{\nu} \mathbf{u}_{T} \cdot \nabla_{T} u_{\nu} d \Gamma\right| \leq C\left(\int_{\Gamma_{1}}|\mathbf{u}|^{2} d \Gamma+\|\mathbf{u}\|_{\mathrm{H}^{1}(\Omega)}^{2}\right) .
$$

With Poincaré's inequality and Korn's inequality, we finally get

$$
\left|\int_{\Gamma_{1}} 2 B h_{\nu} \mathbf{u}_{T} \cdot \nabla_{T} u_{\nu} d \Gamma\right| \leq C E(\mathbf{u}, t)
$$

Observing that the energy function is non-increasing and using (33), (35), we obtain

$$
\begin{aligned}
\left|\int_{S}^{T} \int_{\Gamma_{1}} 2 B h_{\nu} \mathbf{u}_{T}^{\prime} \cdot \nabla_{T} u_{\nu} d \Gamma d t\right| \leq & C E(\mathbf{u}, S) \\
& +\theta \int_{S}^{T}\left\|\mathbf{u}_{T}\right\|_{1}^{2} d t+\frac{C}{\theta} \int_{S}^{T} \int_{\Gamma_{1}}\left|\mathbf{u}^{\prime}\right|^{2} d \Gamma d t .
\end{aligned}
$$

Using boundedness of $\mathbf{h}$, we get

$$
\left|\int_{\Gamma_{1}} 2 B h_{\nu} u_{\nu}^{\prime} \partial_{\nu} u_{\nu} d \Gamma\right| \leq \theta \int_{\Gamma_{1}}\left|\partial_{\nu} u_{\nu}\right|^{2} d \Gamma+\frac{C}{\theta} \int_{\Gamma_{1}}\left|\mathbf{u}^{\prime}\right|^{2} d \Gamma .
$$

Finally, with (28)-(32), (36) and (37), we obtain

$$
\begin{aligned}
\left|\mathcal{I}_{2}\right| \leq & \theta \int_{S}^{T} \int_{\Gamma_{1}}\left(\left|\partial_{\nu} u_{\nu}\right|^{2}+\varepsilon_{T}\left(\mathbf{u}_{T}\right): \varepsilon_{T}\left(\mathbf{u}_{T}\right)+\left|\varepsilon_{S}(\mathbf{u})\right|^{2}\right) d \Gamma d t \\
& +\frac{C}{\theta} \int_{S}^{T} \int_{\Gamma_{1}}\left(|\mathbf{u}|^{2}+\left|\mathbf{u}^{\prime}\right|^{2} d \Gamma d t+C E(\mathbf{u}, S) .\right.
\end{aligned}
$$

Again, we emphasize that, in (38), $\theta$ is a positive number which will be chosen later on and $C$ is a positive constant which does not depend on $\mathbf{u}$.

\section{- End of the proof.}

With (21), (27), (38), we obtain that there exists two positive constants $C_{6}$ and $C_{7}$ such that

$$
\begin{aligned}
C_{1} \int_{S}^{T} E d t \leq & C_{6} E(\mathbf{u}, S)+\frac{C_{7}}{\theta} \int_{S}^{T} \int_{\Gamma_{1}}\left(|\mathbf{u}|^{2}+\left|\mathbf{u}^{\prime}\right|^{2}\right) d \Gamma d t \\
& +2 \theta \int_{S}^{T} \int_{\Gamma_{1}}\left(\varepsilon_{T}(\mathbf{u}): \varepsilon_{T}(\mathbf{u})+\left|\varepsilon_{\nu}(\mathbf{u})\right|^{2}+\left|\varepsilon_{S}(\mathbf{u})\right|^{2}\right) d \Gamma d t \\
& -2 k \mu \int_{S}^{T} \int_{\Gamma_{1}}\left(\varepsilon_{T}(\mathbf{u}): \varepsilon_{T}(\mathbf{u})+\left|\varepsilon_{\nu}(\mathbf{u})\right|^{2}+2\left|\varepsilon_{S}(\mathbf{u})\right|^{2}\right) d \Gamma d t \\
& -k \lambda \int_{S}^{T} \int_{\Gamma_{1}}\left(\operatorname{tr}\left(\varepsilon_{T}(\mathbf{u})\right)+\varepsilon_{\nu}(\mathbf{u})\right)^{2} d \Gamma d t
\end{aligned}
$$


From the relations

$$
\varepsilon_{T}(\mathbf{u})=\varepsilon_{T}\left(\mathbf{u}_{T}\right)+u_{\nu} \partial_{T} \nu=\varepsilon_{T}\left(\mathbf{u}_{T}\right)+\varepsilon_{T}\left(u_{\nu} \nu\right), \quad \text { on } \Gamma,
$$

we have

$$
\begin{aligned}
& \varepsilon_{T}(\mathbf{u}): \varepsilon_{T}(\mathbf{u})= \\
& \quad \varepsilon_{T}\left(\mathbf{u}_{T}\right): \varepsilon_{T}\left(\mathbf{u}_{T}\right)+2 \varepsilon_{T}\left(\mathbf{u}_{T}\right): \varepsilon_{T}\left(u_{\nu} \nu\right)+\varepsilon_{T}\left(u_{\nu} \nu\right): \varepsilon_{T}\left(u_{\nu} \nu\right), \quad \text { on } \Gamma_{1} .
\end{aligned}
$$

Using $\left|\varepsilon_{T}\left(\mathbf{u}_{T}\right): \varepsilon_{T}\left(u_{\nu} \nu\right)\right| \leq \theta \varepsilon_{T}\left(\mathbf{u}_{T}\right): \varepsilon_{T}\left(\mathbf{u}_{T}\right)+(4 \theta)^{-1} \varepsilon_{T}\left(u_{\nu} \nu\right): \varepsilon_{T}\left(u_{\nu} \nu\right)$, we get

$$
\varepsilon_{T}(\mathbf{u}): \varepsilon_{T}(\mathbf{u}) \geq(1-2 \theta) \varepsilon_{T}\left(\mathbf{u}_{T}\right): \varepsilon_{T}\left(\mathbf{u}_{T}\right)+\left(1-\frac{1}{2 \theta}\right) \varepsilon_{T}\left(u_{\nu} \nu\right): \varepsilon_{T}\left(u_{\nu} \nu\right), \text { on } \Gamma_{1} .
$$

Since $\varepsilon_{T}\left(u_{\nu} \nu\right): \varepsilon_{T}\left(u_{\nu} \nu\right) \leq C \int_{\Gamma_{1}}\left|u_{\nu}\right|^{2} d \Gamma$, we deduce from (39) that there exists $C_{8}>0$ such that

$$
\begin{array}{rl}
C_{1} \int_{S}^{T} E & d t \leq C_{6} E(\mathbf{u}, S)+\frac{C_{8}}{\theta} \int_{S}^{T} \int_{\Gamma_{1}}\left(|\mathbf{u}|^{2}+\left|\mathbf{u}^{\prime}\right|^{2}\right) d \Gamma d t \\
& +2 \theta \int_{S}^{T} \int_{\Gamma_{1}}\left(\varepsilon_{T}(\mathbf{u}): \varepsilon_{T}(\mathbf{u})+\left|\varepsilon_{\nu}(\mathbf{u})\right|^{2}+\left|\varepsilon_{S}(\mathbf{u})\right|^{2}\right) d \Gamma d t \\
& -2 k \mu \int_{S}^{T} \int_{\Gamma_{1}}\left((1-2 \theta) \varepsilon_{T}(\mathbf{u}): \varepsilon_{T}(\mathbf{u})+\left|\varepsilon_{\nu}(\mathbf{u})\right|^{2}+2\left|\varepsilon_{S}(\mathbf{u})\right|^{2}\right) d \Gamma d t
\end{array}
$$

Then, for $\theta>0$ small enough, we can find a positive constant $C_{9}$ such that

$$
C_{1} \int_{S}^{T} E d t \leq C_{6} E(\mathbf{u}, S)+C_{9} \int_{S}^{T} \int_{\Gamma_{1}}\left(|\mathbf{u}|^{2}+\left|\mathbf{u}^{\prime}\right|^{2}\right) d \Gamma d t .
$$

Thanks to Proposition 3 and Lemma 5, there exists $C_{10}>0$ such that, for every $\eta>0$,

$$
C_{1} \int_{S}^{T} E d t \leq \frac{C_{10}}{\eta} E(\mathbf{u}, S)+\eta \int_{S}^{T} E d t .
$$

Hence, for $\eta$ small enough, we conclude the proof of Theorem 1 by applying Lemma 1 and by setting $\omega=\frac{\left(C_{1}-\eta\right) \eta}{C_{10}}$.

Now, we can observe that above constants (especially $C_{1}, C_{10}, \eta$ do not depend on the strong solution $\mathbf{u}$ of (2). Hence, by a density argument, this result can be extended to a weak solution of $(2)$.

\section{Application: proof of Theorem 2}

We show that Theorem 1 can be applied with the following vector field.

$$
\mathbf{h}(\mathbf{x})=\left(\mathbf{x}-\mathbf{x}_{0}\right)+\rho \widetilde{\mathbf{h}}(\mathbf{x}),
$$


where $\rho$ is some positive constant and $\widetilde{\mathbf{h}} \in\left(\mathcal{C}^{1}(\bar{\Omega})\right)^{3}$ is such that

$$
\widetilde{\mathbf{h}}=0, \quad \text { on } \Gamma_{0}, \quad \widetilde{\mathbf{h}}=\nu, \quad \text { on } \Gamma_{1} .
$$

- h satisfies (4). Indeed we have

$$
\begin{array}{ll}
\mathbf{h}(\mathbf{x}) \cdot \nu(\mathbf{x})=\left(\mathbf{x}-\mathbf{x}_{0}\right) \cdot \nu(\mathbf{x}) \leq 0, & \text { if } \mathbf{x} \in \Gamma_{0} \\
\mathbf{h}(\mathbf{x}) \cdot \nu(\mathbf{x})=\left(\mathbf{x}-\mathbf{x}_{0}\right) \cdot \nu(\mathbf{x})+\rho>0, & \text { if } \mathbf{x} \in \Gamma_{1}
\end{array}
$$

- h satisfies (6). We have

$$
\int_{\Omega} \sigma_{\imath \jmath}(\mathbf{v}) h_{k, \jmath} \mathbf{v}_{\imath, k} d \mathbf{x}=\int_{\Omega} \sigma_{\imath \jmath}(\mathbf{v}) \xi_{\imath, \jmath} d \mathbf{x}+\rho \int_{\Omega} \sigma_{\imath \jmath}(\mathbf{v}) \widetilde{h}_{k, \jmath} \mathbf{v}_{\imath, k} d \mathbf{x} .
$$

Using Korn's inequality, we can build a constant $C(\widetilde{\mathbf{h}})>0$ such that

$$
\left|\int_{\Omega} \sigma_{\imath \jmath}(\mathbf{v}) \widetilde{h}_{k, \jmath} \mathbf{v}_{\imath, k} d \mathbf{x}\right| \leq C(\widetilde{\mathbf{h}}) \int_{\Omega} \sigma(\mathbf{v}): \varepsilon(\mathbf{v}) d \mathbf{x},
$$

and

$$
\int_{\Omega} \sigma_{\imath \jmath}(\mathbf{v}) h_{k, \jmath} \mathbf{v}_{\imath, k} d \mathbf{x} \geq(1-\rho C(\widetilde{\mathbf{h}})) \int_{\Omega} \sigma(\mathbf{v}): \varepsilon(\mathbf{v}) d \mathbf{x} .
$$

We choose $\alpha=1-\rho C(\widetilde{\mathbf{h}})$ and get $\alpha>0$ for $\rho$ small enough.

Now one can easily show that all conditions in (6) are satisfied if

$$
\rho<\min \left(\frac{1}{C(\widetilde{\mathbf{h}})}, \frac{2}{2 C(\widetilde{\mathbf{h}})+\max _{\bar{\Omega}}(\operatorname{div}(\widetilde{\mathbf{h}}))-\min _{\bar{\Omega}}(\operatorname{div}(\widetilde{\mathbf{h}}))}, \frac{3}{\left|\min _{\bar{\Omega}}(\operatorname{div}(\widetilde{\mathbf{h}}))\right|}\right) .
$$

This completes the proof.

Acknowledgements. We would like to thank very much the referee for his remarks and comments which allow us to improve and clarify some points in our original version.

\section{References}

[1] F. Alabau, V. Komornik, Observabilité, controlabilité et stabilisation frontière du système d'élasticité linéaire, C.R. Acad. Sci. Paris, série I Math. 324, 1997, 519-524.

[2] F. Alabau, V. Komornik, Boundary observability, controllability, and stabilization of linear elastodynamic systems, SIAM Journal on Control and Optimization, Vol. 37, Nr 2, 1999, 521-542.

[3] R. Bey, A. Heminna, J.-P. Lohéac, Stabilisation frontière du système de l'élasticité. Nouvelle approche, C.R. Acad. Sci. Paris, série I Math. 330, 2000, 563-566. 
[4] A. Guesmia, Observability, controllability and boundary stabilization of some linear elasticity systems, Acta Scientiarum Mathematicarum, Vol. 64, Nr 1-2, 1998, 109-120.

[5] A. Guesmia, Existence globale et stabilisation frontière non linéaire d'un système d'élasticité, Portugalice Mathematica, Vol. 56, Nr 3, 1999, 361-379.

[6] A. Guesmia, On the decay estimates for elasticity systems with some localized dissipations, Asymptotic Analysis, Vol. 22, Nr 1, 2000, 1-14.

[7] M.A. Horn, Implications of sharp trace regularity results on boundary stabilization of the system of linear elasticity, Journal of Mathematical Analysis and Applications, 223, 1998, 126-150.

[8] V. Komornik, Exact controllability and stabilization; the multiplier method, MassonJohn Wiley, Paris, 1994.

[9] J.E. Lagnese, Boundary stabilization of linear elastodynamic systems, SIAM J. Control Opt., 21, 1983, 968-984.

[10] J.E. Lagnese, Uniform asymptotic energy estimates for solutions of the equations of dynamic plane elasticity with nonlinear dissipation at the boundary, Nonlinear Anal. TMA, 16, 1991, 35-54.

[11] I. Lasiecka, R. Triggiani, Uniform exponential energy decay of wave equations in a bounded region with $L_{2}\left(0, \infty ; L_{2}(\Gamma)\right)$-feedback control in the Dirichlet boundary conditions, Journal of Differential Equations, 66, 1987, 340-390.

[12] K. Lemrabet, Étude de divers problèmes aux limites de Ventcel d'origine physique ou mécanique dans des domaines non réguliers, Thesis, U.S.T.H.B., Alger, 1987.

[13] J.-L. Lions, Contrôlabilité exacte, perturbation et stabilisation de systèmes distribués, Masson, Paris, 1986.

[14] J.-L. Lions, E. Magenes, Problèmes aux limites non homogènes et leurs applications, Dunod, Paris, 1968.

[15] R. Valid, La mécanique des milieux continus et le calcul des structures, Eyrolles, Paris, 1977. 